
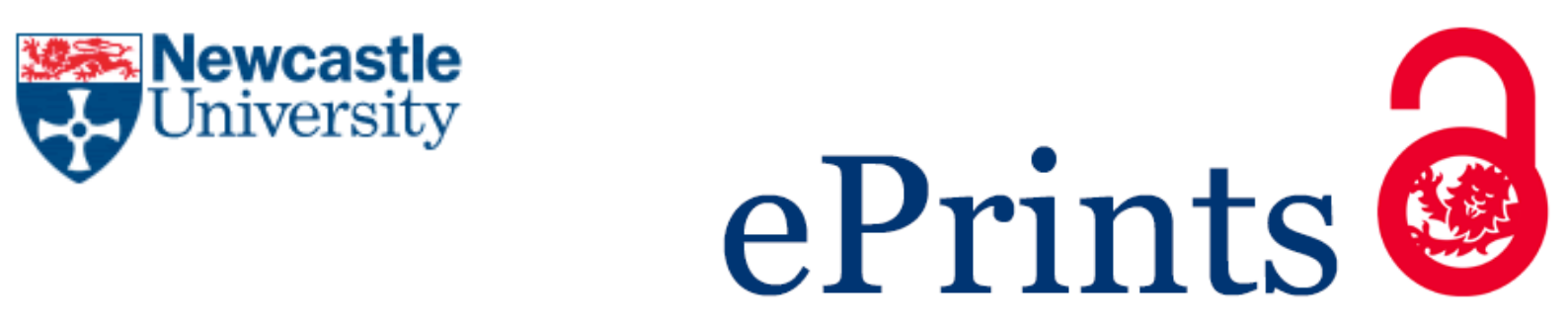

Abdelkareem AE, Sharif BS, Tsimenidis CC.

Adaptive time varying doppler shift compensation algorithm for OFDM-based underwater acoustic communication systems.

Ad Hoc Networks 2016, 45, 104-119

Copyright:

(C) 2016. This manuscript version is made available under the CC-BY-NC-ND 4.0 license

DOI link to article:

http://dx.doi.org/10.1016/j.adhoc.2015.05.011

Date deposited:

$20 / 09 / 2017$

Embargo release date:

04 July 2016

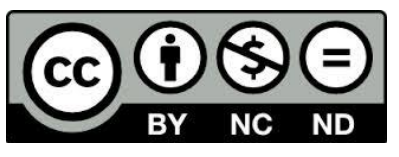

This work is licensed under a

Creative Commons Attribution-NonCommercial-NoDerivatives 4.0 International licence 


\title{
Adaptive Time Varying Doppler Shift \\ Compensation Algorithm for OFDM-based \\ Underwater Acoustic Communication Systems
}

\author{
A. E. Abdelkareem, B. S. Sharif, Senior Member, IEEE, \\ and C. C. Tsimenidis, Member, IEEE \\ Email: a.e.abdelkareem@tu.edu.iq
}

Mobile: (+964) 7705802111

\begin{abstract}
This paper presents an adaptive approach to address the two main problems associated with the time varying Doppler shift, the first being the acceleration effects on the cyclic-prefix (CP) correlation and the second, the effect of a sudden change in the velocity direction between packets on the entire orthogonal frequency division multiplexing (OFDM) symbols. In addition, this paper considers the residual Doppler shift or carrier frequency offset (CFO) that was estimated iteratively within a range according to a design based on the sub-carrier spacing using pilots, which are basically utilized for the purpose of channel estimation. Furthermore, the proposed algorithm adopts three estimations of the symbol timing offset. These estimations are centroid-based localization over an anticipated $\mathrm{CP}$ window, first order expectation and autocorrelation of the received CP with its replica. Subsequently, a penalization algorithm is applied in order to drop the anomalous parameter among them. Therefore, the consequences of the inflection point that accompanies the abrupt change in the velocity are mitigated and a reliable time varying Doppler shift is obtained. This Doppler shift is fine tuned in an iterative manner. Compared with the block-based Doppler compensation approach, the proposed technique works with variable speed during packet duration. In addition, it exploits the available bandwidth more efficiently by utilizing a single preamble of linear-frequency-modulation (LFM) to detect the start of the packet. The proposed receiver was evaluated through simulations and sea trials conducted over $500 \mathrm{~m}$ and $1000 \mathrm{~m}$ channel ranges. In simulations, a model was designed to imitate the time varying Doppler shift with two scenarios (expansion/compression) in combination with a various multipath delay spread. The simulation results confirm that the proposed system accommodates an acceleration up to $\pm 1 \mathrm{~m} / \mathrm{s}^{2}$ during the packet time.
\end{abstract}

Index Terms

Time varying Doppler, weighted centroid localization, OFDM, underwater acoustic communication, bit interleaved coded modulation, iterative decoding.

\section{INTRODUCTION}

Several time-domain receivers that adopt coherent modulation with an emphasis on channel equalization in order to increase communication reliability have been suggested. However, the time varying doubly-spread characteristic of 
the underwater acoustic (UWA) channel requires a highly complex equalizer. Recently, an alternative low-complexity, high-speed multi-carrier communication system, in the form of OFDM, has attracted considerable interest in the field of underwater acoustic communication (UWAC). This is mainly due to its simplicity of operation by means of the fast Fourier transform (FFT) for the purpose of modulation/demodulation. This system deals with the frequency selectivity of the channel by dividing the broadband data into parallel narrowband channels. Additionally, in a delaydispersive environment, adopting a CP of a length greater than the maximum delay spread provides an excellent way to assure the orthogonality of the carriers. However, propagation is considered to be time-selective due to the Doppler shift in which one sub-carrier may introduce ICI to the adjacent sub-carriers [1]. The Doppler shift sensitivity is proportional to the OFDM symbol duration; therefore even slightly moving platforms can cause serious impairments as far as synchronization is concerned.

Previous studies in the field of UWAC have addressed several approaches for synchronization in the presence of Doppler distortion. As far as multi-carrier transmission is concerned, the authors in [2]-[4] have addressed the performance of the OFDM systems under the Doppler effects and investigated the Doppler scale methods, respectively. Researchers in [5] employed the principle in [6] and null sub-carriers for the purpose of re-sampling factor estimation and residual Doppler compensation, respectively. Although these algorithms do attain precise estimation by adopting preamble and post-amble, the bandwidth utilization factor is compromised. A point estimate of the Doppler shift is adopted in [7]; therefore it is suitable for situations where the Doppler shift stays constant or varies slowly during the packet time. The concept in [8] was extended to work in UWAC by [9] with an iterative cyclic prefix correlation. To estimate the Doppler shift, the author employed the symmetry of the guard interval with its replica. This parameter is estimated iteratively, depending on the peak location and its phase with respect to the new sampling interval; therefore it is a computationally expensive search.

A number of previous studies have based their criteria for Doppler-shift and CFO estimation on utilizing signal space and statistics. For instance, the authors in [10] have used maximal likelihood estimation (MLE) and ESPRIT to estimate both CFO and Doppler shift in wideband OFDM, while in [11], HTLS (Hankel Total Least Square) has been used for joint channel and Doppler estimation. The system requires no estimation of the CFO, and there is no need to re-sample the signal. An extension to [7] has been suggested by [12] for symbol by symbol Doppler estimation. This method adopts marginal maximum likelihood estimation (MMLE) to track the Doppler variation between symbols. Despite its precise estimation, MLE has a number of problems in terms of hardware implementation, where it necessitates high complexity.

Although re-sampling the signal removes the Doppler shift, a major problem with its residual or CFO is the destruction of the orthogonality of the sub-carriers due to the resulting inter-carrier interference (ICI). A considerable amount of literature on combating ICI has been published. These studies [13]-[16] have presented conclusions that mitigating ICI will result in successful communication.

All the aforementioned papers assume that the Doppler shift is constant during the symbol period and all paths have equal Doppler shift; therefore re-sampling the signal with a unique time scaling factor is valid and a symbol by symbol approach works effectively. A recent study by [17] has highlighted the need to estimate the optimal time 


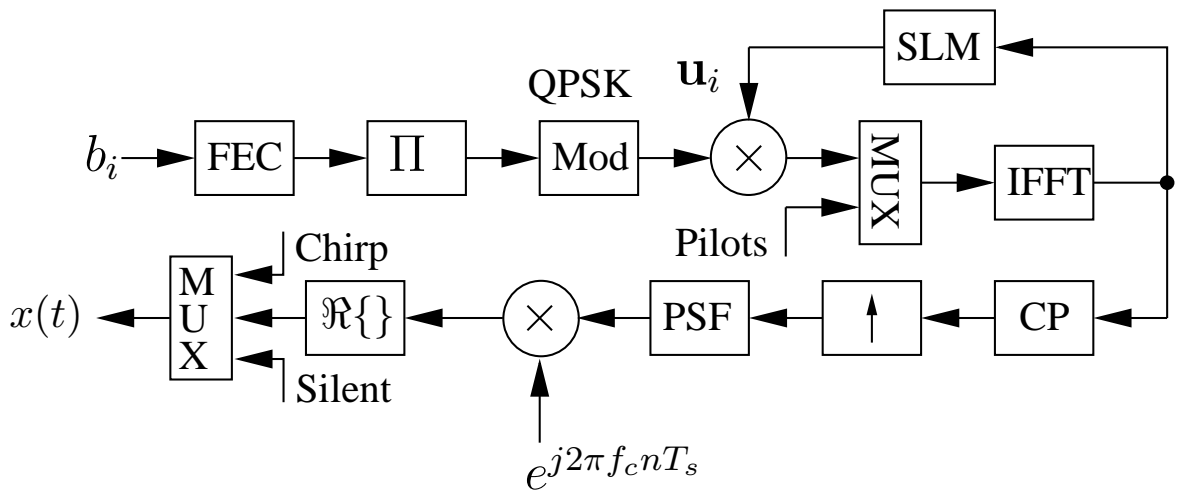

Fig. 1. Proposed transmitter structure, where the operator $\Re$ represents the real part of the signal.

scaling factor in a multipath channel of different Doppler shift in each path. However, in our proposed method, it is assumed that the channel variation is mainly caused by the motion of both transmitter and receiver, leading to a significant time varying Doppler shift. Consequently, this will create acceleration that may exceed $1 \mathrm{~m} / \mathrm{s}^{2}$ due to speed alterations; therefore, ignoring this effect yields a significant ICI. In this paper, the acceleration is considered and the Doppler shift is assumed to be changing linearly during the symbol time, but constant in all paths. This variation is dealt with by measuring the time expansion/compression frequently within a fraction of a sample period and then compensating the Doppler by means of an efficient 4th order Lagrange interpolation.

In this paper, the proposed algorithm adopts an iterative approach in estimating the first order moment that results in minimum phase and decoding errors. A cyclic redundancy check (CRC) is employed to detect bit errors after decoding. Additionally, the iterations rely on the criteria of minimum phase error estimation to compensate the residual Doppler shift. In the proposed technique, the estimation errors are subject to penalization by enabling a learning and punishment (LP) action to fine tune the estimated shift in samples iteratively. Only the minimum phase error which accompanies the $i$ th iteration is chosen with its associated time expansion and/or compression, therefore an accurate Doppler shift is obtained. In learning mode (LM), the acceleration of the previous packet is observed to designate an adaptive expectation range (ER), whereas the punishment mode (PM) drops an out of range estimation.

The rest of this paper is organized as follows. In section II, the proposed OFDM system and channel model are introduced. The acceleration effects are demonstrated in section III. In section IV, the proposed adaptive receiver is presented. In section V, system design, simulation and experimental results are presented. Finally, section VI draws the conclusions.

\section{SYSTEM AND CHANNEL MODEL}

The proposed system contains the transmitter depicted in Fig.1. At each instant $i$, the encoder receives a vector of information bits $b_{i}$ of length $K_{d}$ at its input to produce a binary code of length $K_{c}=K_{d} / R_{c}$ encoded bits, where $R_{c} \in(0,1]$ is the coding rate of the nonsystematic convolutional (NSC) code. The coded bits are permuted 
by a random interleaver, then converted, in groups of $m$ successive bits, into alphabet symbols of constellation size $M=2^{m}$. This mapping operation generates a sequence of $N_{d}=K_{c} / m: \mathbf{s}=\left\{s_{0} \ldots s_{N_{d}-1}\right\}$, where $s_{i} \in \mathbb{C}$ and $\mathbb{C}$ denotes the set of complex symbols. Subsequently, in the OFDM symbol to be constructed, pilot symbols of phase shift keying (PSK) with unit amplitude are embedded with the data symbols in a comb method. These pilot symbols are used for the purpose of estimating channel response at the receiver. A PAPR reduction is introduced using the SLM technique [18]. To implement this technique, a sequence of phases $\mathbf{U}$ are added in the transmitted signal to be multiplied by the input data sequences and the symbol sequence of minimum PAPR is selected for transmission. The resulting OFDM symbol, containing $N_{p}$ pilots and $N_{d}$ data-bearing sub-carriers, where $N_{d}+N_{p}=N_{c}$, is then modulated by an IFFT of size $N_{c}$ and the last samples are copied and prefaced to the symbol to form the CP-OFDM frame. The guard interval of length $N_{g}$ is chosen to be longer than the channel dispersion time in order to minimize the inter-symbol interference (ISI). The resulting frame is pulse shaped, using a pulse shape filter (PSF), and then up-converted using carrier modulation. Let $T_{d}$ denote the OFDM symbol duration and $T_{g}$ the guard interval. The total OFDM frame duration is $T=T_{d}+T_{g}$. Let $f_{n}=f_{c}+n \Delta_{f}$, being the carrier frequency corresponding to each of the sub-carriers of the OFDM spectrum, where $\Delta_{f}=1 / T_{d}$ is the frequency separation between alternate sub-carriers and $f_{c}$ is the carrier frequency, so the bandwidth is $B=N_{c} \Delta_{f}$. The time-domain representation of the $i$ th OFDM symbol is given by

$$
\begin{gathered}
x_{i}(t)=\frac{1}{\sqrt{N_{\mathrm{c}}}} \sum_{n \in \mathcal{I}} d_{i}(n) u_{i}^{o p t}(n) e^{j 2 \pi \frac{n}{T_{d}}\left(t-T_{g}-i T\right)} * p_{\mathrm{rc}}(t-i T), \\
\text { for } i T \leq t<(i+1) T,
\end{gathered}
$$

where $d_{i}(n)$ is the symbol transmitted over the $n$th sub-carrier, $\mathbf{U}^{o p t}$ is the optimum phase set $\left[u_{i}(1), u_{i}(2), \ldots . u_{i}(n)\right]$ for lower PAPR with $u_{i}(n)=e^{j \varphi_{n}}, \varphi_{n} \in[0,2 \pi], \mathcal{I}$ denotes the set of modulated sub-carriers and $p_{r c}(t-i T)$ is the pulse shaping filter, which is realized as an up-sampled raised cosine FIR filter. An equivalent passband model of (1) is

$$
\begin{aligned}
x_{i}(t) & =\Re\left\{e^{j 2 \pi f_{c} t} \sum_{i=0}^{\infty} \frac{1}{\sqrt{N_{\mathrm{c}}}} \sum_{n \in \mathcal{I}} d_{i}(n) u_{i}^{o p t}(n) e^{j 2 \pi \frac{n}{T_{d}}\left(t-T_{g}-i T\right)} * p_{\mathrm{rc}}(t-i T)\right\}, \\
& =\Re\left\{\sum_{i=0}^{\infty} \frac{1}{\sqrt{N_{\mathrm{c}}}} \sum_{n \in \mathcal{I}} d_{i}(n) u_{i}^{o p t}(n) e^{j 2 \pi f_{n} t} e^{j 2 \pi \frac{n}{T_{d}}\left(T_{g}-i T\right)} * p_{\mathrm{rc}}(t-i T)\right\},
\end{aligned}
$$

It is assumed that the signal is transmitted over a multipath fading channel

$$
h(\tau, t)=\sum_{l=0}^{L-1} h_{l}(t) \delta\left[\tau-\tau_{l}(t)\right],
$$

where $\left\{h_{l}(t)\right\}$ are the path amplitudes, $\left\{\tau_{l}(t)\right\}$ are the time varying path delays and $L$ is the total number of paths. As in [19], we assume the path delay $\tau_{l}$ and the gains $h_{l}$ are constant over the frame duration $T$.

1) For perfect OFDM synchronization, and providing that the maximum delay spread is within the guard interval, the received passband signal can be written as 


$$
\begin{aligned}
\tilde{r}(t)= & \Re \sum_{i=0}^{\infty} \frac{1}{\sqrt{N_{\mathrm{c}}}} \sum_{n \in \mathcal{I}} d_{i}(n) u_{i}^{o p t}(n) e^{j 2 \pi f_{n} t} \\
& \left.\times \sum_{l=0}^{L-1} h_{l} * p_{r c}\left(t-\tau_{l}\right) e^{-j 2 \pi f_{n} \tau_{l}}\right\}+\tilde{w}_{i}(t),
\end{aligned}
$$

where $w_{i}(t)$ is a white Gaussian noise with variance $\sigma^{2}$.

$$
r(t)=\sum_{l=0}^{L-1} x\left[\left(1 \pm \frac{v}{c}\right) t-\tau_{l}\right]
$$

where $v$ denotes the induced speed due to the mobility of the transmitter and/or receiver and $c$ is the acoustic propagation speed of $1500 \mathrm{~m} / \mathrm{s}$. The $(+)$ sign indicates an expansion of the signal since the distance is increased and vice versa. It is assumed that all paths have a similar $\Delta$; therefore,

2) when the Doppler shift is present, the received signal in (4) can be rewritten as

$$
\begin{aligned}
\tilde{r}(t)= & \Re\left\{\sum_{i=0}^{\infty} \frac{1}{\sqrt{N_{\mathrm{c}}}} \sum_{n \in \mathcal{I}} d_{i}(n) u_{i}^{o p t}(n) e^{j 2 \pi f_{n}(1+\Delta) t}\right. \\
& \left.\cdot \sum_{l=0}^{L-1} h_{l} * p_{r c}\left((1+\Delta) t-\tau_{l}\right) e^{-j 2 \pi f_{n} \tau_{l}}\right\}+\tilde{w}_{i}(t),
\end{aligned}
$$

Based on the assumption that the speed of the motion changes linearly during the $i$ th OFDM symbol interval $t \in[i T, T(i+1))$, the Doppler shift is varied with time; therefore the constant $\Delta=\frac{v}{c}$ does not hold to accommodate this variation and it should be replaced by $\Delta(t)$. Thus, the time varying Doppler shift can be modeled as

$$
\Delta(t)=\mp \frac{v(t)}{c},
$$

where $v(t)$ represents the speed variation during the symbol time.

3) Based on (7), the received passband signal in (6) with a time varying Doppler shift can be rewritten as,

$$
\begin{aligned}
\tilde{r}(t)= & \Re\left\{\sum_{i=0}^{\infty} \frac{1}{\sqrt{N_{\mathrm{c}}}} \sum_{n \in \mathcal{I}} d_{i}(n) u_{i}^{o p t}(n) e^{j 2 \pi f_{n}(1+\Delta(t)) t}\right. \\
& \left.\cdot \sum_{l=0}^{L-1} h_{l} p_{r c}\left[(1+\Delta(t)) t-\tau_{l}\right] e^{-j 2 \pi f_{n} \tau_{l}}\right\}+\tilde{w}_{i}(t),
\end{aligned}
$$

and its corresponding complex baseband signal model can be written as

$$
r(t)=\sum_{i=0}^{\infty} \sum_{n \in \mathcal{I}} H_{i}(n) d_{i}(n) u_{i}^{o p t}(n) e^{j 2 \pi n \Delta_{f} t} e^{j 2 \pi \Delta(t) f_{n} t}+w_{i}(t),
$$

where $H_{i}(n)$ is the channel transfer function of the $i$ th symbol at $n$th sub-carrier with a time varying Doppler-shift that can be written as

$$
H_{i}(n)=\sum_{l=0}^{L-1} h_{l} e^{-j 2 \pi f_{n} \tau_{l}} p_{r c}\left[1+\Delta(t) t-\tau_{l}-i T\right]
$$


As referred to in [20], it is clearly shown in (9) that the effect of the Doppler shift on the received signal is twofold. Firstly, it scales the received OFDM frame duration $T$ by a factor of $1+\Delta(t)$, yielding sampling frequency errors that result in a symbol timing error [22]. Secondly, there is a time varying CFO.

\section{A. Sampling frequency errors}

In discrete time, the sampled transmitted signal $x\left[k T_{s}\right]$ in (5) is equivalent to a scaling of the sampling period (interpolation or decimation)

$$
\tilde{r}\left[k T_{s}\right]=x\left[k(1 \mp \Delta(t)) T_{s}-\tau_{l}\right]
$$

where $k$ is an integer, and $T_{s}$ and $\tilde{r}\left(k T_{s}\right)$ are the sampling period and Doppler shifted received sampled signals respectively.

The bidirectional effect of the Doppler shift causes symbol timing errors, which are increased or decreased proportionally to $\Delta(t)$. To align the symbol within its period, samples should be removed if $(\Delta>0)$ or added if $(\Delta<0)$ at regular intervals [21].

Let $\pm \phi$ be the deviation of samples of the received sequence for each OFDM symbol due to the speed change; the sampling period results in expansion or compression of the samples' length, hence the Doppler-shifted received frame's length is modeled by

$$
L_{f}^{\prime}=\left(L_{f} \mp \phi\right)
$$

where $L_{f}=\frac{N_{c}}{B \cdot T_{s}}$ represents the transmitted passband samples' length. It is apparent that $L_{f}$ is only affected by $T_{s}$ and any expansion/compression in the timescale will result in $\phi$. Therefore, (12) is implicitly equivalent to (11). To remove both CFO and symbol shift, an inverse time scaling of the received (compressed/expanded) signal should be achieved providing that the amount of Doppler shift $\Delta(t)$ is known. This is equivalent to changing the sampling rate of the passband signal by $1+\Delta(t)$ in discrete-time processing. From (12), it can be inferred that increasing or decreasing the length of samples is equivalent to adjusting the sampling frequency $f_{s}$ by the same Doppler shift $1+\Delta(t)$; thus (11) is rewritten as

$$
\tilde{r}[k]=x\left[\frac{k(1 \mp \Delta(t))}{f_{s}^{\prime}}-\tau_{l}\right],
$$

where $f_{s}^{\prime}=f_{s}(1 \mp \Delta(t))$. By substituting $f_{s}^{\prime}$ in (13), $\tilde{r}[k]=x[k]$, the signal received is then in conformity with the transmitted signal.

\section{B. Carrier frequency offset errors}

The factor $e^{j 2 \pi \Delta(t) f_{n} t}$ in the received signal in (9) represents a time varying CFO, where $\Delta(t) f_{n}=\Delta(t) f_{c}+$ $\Delta(t) n \Delta_{f}$. The CFO $(\epsilon)$ is due to the residual Doppler shift. It is destructive because it deviates the sub-carrier spacing $\Delta_{f}$ and introduces ICI, which must be removed prior to the FFT to design an optimum receiver [22]. The re-sampling process removes the Doppler shift and converts the wideband system into narrowband. However, the residual Doppler shift produced by the fractional part of the time expansion/compression degrades the receiver. 


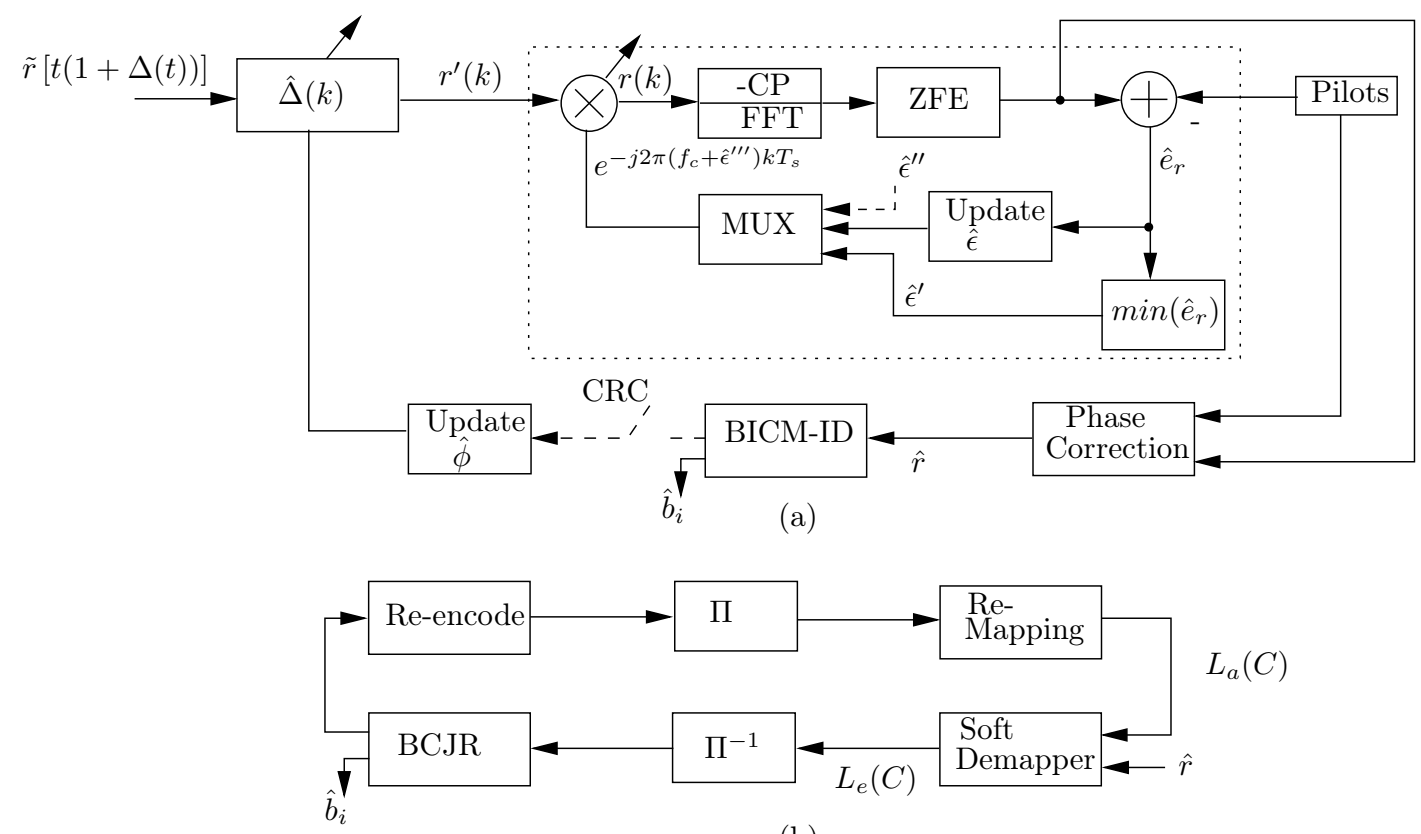

(b)

Fig. 2. Receiver structure

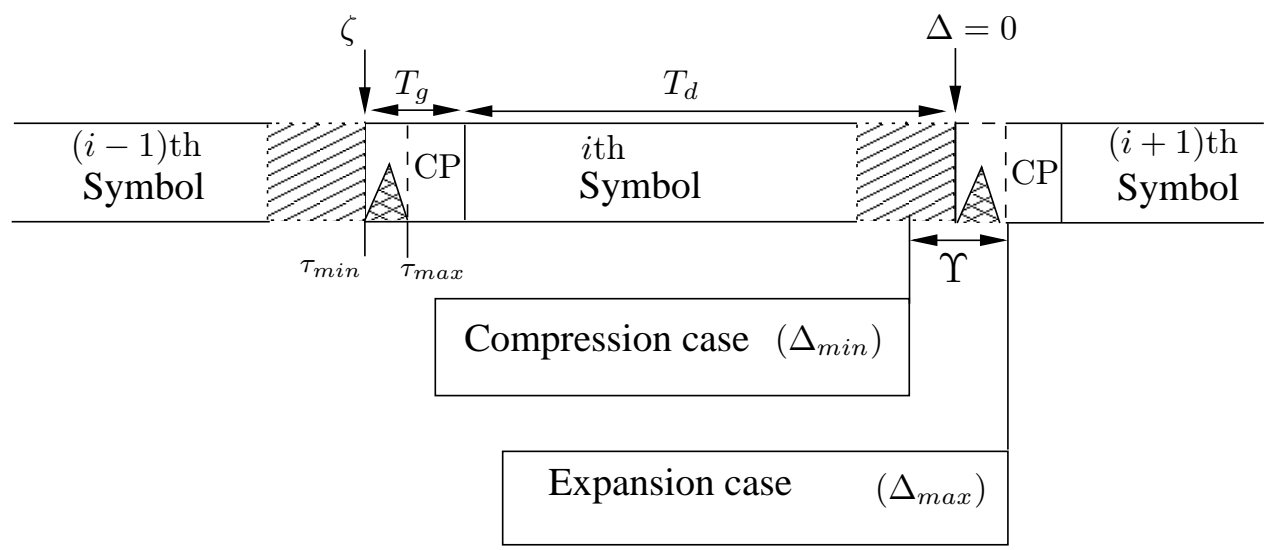

Fig. 3. OFDM symbol structure due to Doppler effects, where $\Upsilon$ represents an anticipated window to observe the time expansion and/or compression.

\section{AdAPTIVE OFDM RECEIVER STRUCTURE}

The receiver structure is comprised of an acquisition stage, an estimation of the cyclic prefix position (symbol timing), an adaptive Doppler shift estimation and compensation, and channel decoding. The receiver block diagram is presented in Fig. 2. This receiver deals with three different cases of an OFDM symbol subject to Doppler shift as shown in Fig. 3. The first case is where there is no Doppler shift or $\Delta=0$. In this case, the OFDM symbol coincides with the exact timing, preserving the orthogonality among sub-carrier frequency components. In 
the compression case, the symbol time is reduced and the sampling frequency must be increased to compensate for the Doppler shift whereas in the expansion case, the symbol time is increased and the sampling frequency is reduced. In addition, the received signal within the FFT window contains a part of the current OFDM symbol and part of the proceeding OFDM symbol. This causes an ISI and an ICI, which implies that the orthogonality has been compromised. In open-loop receivers, the Doppler shift is approximated based on one-shot estimation. The iterative receiver, instead of depending on a single estimation of the centroid-based localization and linear prediction to estimate the Doppler shift, combines conventional autocorrelation and then averaging based on three estimations. Furthermore, the pilot has been utilized for phase error detection and correction in addition to channel estimation. The proposed system adopts an iterative estimation of the first order moment that results in minimum phase and decoding errors. In a practical communication system, there is a CRC to detect bit errors after decoding and an action such as retransmission or repeat decoding is taken. Additionally, the iterations rely on the criteria of minimum phase error estimation to compensate the residual Doppler shift. In the proposed technique, the estimation errors are subject to penalization by enabling a LP action to fine tune $\phi$ iteratively. Only the minimum phase error which accompanies the $i$ th iteration is chosen with its associated $\phi$, therefore an accurate Doppler shift is obtained. In LM, the acceleration of the previous packet is observed to designate an adaptive ER, whereas the PM drops an out of range estimation.

\section{A. Signal acquisition}

The frontend of the receiver consists of bandpass filter (BPF) of carrier frequency $f_{c}$ to remove the unwanted low frequencies due to the ambient noise. The subsequent stage is to identify the start of the packet through the finite impulse response (FIR)-correlator with the chirp signal. The resulting peak signal represents the coarse estimation of the packet starting point $\zeta$, which contains the received passband Doppler-shifted samples and is directed to the symbol synchronization stage.

\section{B. Estimation of symbol timing expansion/compression}

By considering the effect of acceleration on the chirp correlation is small, then in the case of multiple OFDM symbols within a packet, the symbol timing error in each OFDM block is accumulated with acceleration during the packet time. Hence, adopting a single estimation of the synchronization point $\zeta$ for the whole packet is no longer accurate. Therefore, in order to mitigate the acceleration effect on the symbol timing error, $\zeta$ needs to be fine tuned. Performing the fine tuning necessitates updating the position of $\zeta$ after each symbol time. Let $m, i$ denote the range of the timing offset around the leading and the trailing edge during the OFDM symbol, respectively. It follows that a two dimensional timing function is written as

$$
\begin{aligned}
& \Lambda(m, i) \triangleq\left|\sum_{n=0}^{N_{g}-1} r(\zeta+m+n) r(\zeta+n+N+i)^{*}\right| \\
& m \in\{-W / 2 \cdots W / 2\} ; i \in\left\{-\Upsilon^{\prime} / 2 \cdots \Upsilon^{\prime} / 2\right\},
\end{aligned}
$$


where, $W$ corresponds to the region around the synchronization point $\zeta$ and $\Upsilon^{\prime}$ represents the region around the tail of the OFDM symbol. Then, $\hat{\theta}_{m, i}$ can be estimated from obtaining the maximum peak of the multiplication and it can be written as

$$
\begin{aligned}
& \hat{\theta}_{m, i}=\arg \max \max _{m, i} \Lambda(m, i) \\
& m \in\{-W / 2 \cdots W / 2\} ; i \in\left\{-\Upsilon^{\prime} / 2 \cdots \Upsilon^{\prime} / 2\right\},
\end{aligned}
$$

and the fine tuned $\zeta^{\prime}$ is obtained. The implementation of this fine tuning algorithm of the coarse packet synchronization can be summarized as follows:

1) compute the coarse packet synchronization point $\zeta$ which represents the time position of the maximum peak of the chirp correlation,

2) compute the timing function $\Lambda(m, i)$ for $m \in[-W / 2, W / 2], i \in\left[-\Upsilon^{\prime} / 2, \Upsilon^{\prime} / 2\right]$,

3) choose the maximum of $\Lambda(m, i)$ as the estimated packet timing offset,

4) update $\zeta$ to be fine tuned which is given as

$$
\zeta^{\prime}=\zeta+\hat{\theta}_{m, i}
$$

It should be noted that a two dimensional search (i.e. $m$ and $i$ ) is included in the proposed timing function $\Lambda(m, i)$. This is the main difference from the single synchronization point estimation in [23], where only coarse estimation of the packet synchronization point is adopted. The first search parameter is $m$, corresponding to the first search region in the range around the coarse synchronization point $\zeta$. Meanwhile, the second search parameter is $i$, corresponding to the range in the region around the tail of the OFDM symbol which yields the expected Doppler shift. Once the fine tuned $\zeta^{\prime}$ is obtained, the subsequent stage is the estimation of the first order moment $\hat{\phi}$. In existing techniques, [8] and [23], due to the acceleration and the inherent ISI, there is a fluctuation in the maximum of the timing function and the channel conditions have a direct effect on this maximum. Therefore, centroid-based localization is adopted to estimate $\hat{\phi}$, because it reduces the position uncertainty caused by the fading channel, and the search range is built on the fine tuned $\zeta^{\prime}$, which can be written as

$$
r_{D} \in\left[\zeta^{\prime}+N-\left(\frac{\Upsilon}{2}\right)+i, \zeta^{\prime}+N+N_{g}-\left(\frac{\Upsilon}{2}\right)+i\right],
$$

and the centroid-based first order moment $\hat{\phi}_{l}$ is given as in [23].

Fine tuning of the coarse symbol timing facilitates an alternative approach to estimating the first order moment of the correlation lag. The suggested approach here aims to increase the confidence of estimation by considering the first order moment that results from two correlation lags. The first correlation lag is estimated by means of centroid-based localization, in accordance with the anticipated window mentioned earlier. This type of correlation gives an accurate indication of the fractional part of the time-scale expansion/compression. However, the centroidbased localization is severely affected due to the velocity perturbation. This perturbation degrades the estimation performance of the timing function and ultimately $\hat{\phi}_{l}$. Therefore, an alternative approach has been adopted by involving another estimation point based upon full correlation of the received $\mathrm{CP}$ with its replica. Adding of this correlation is derived upon the idea of increasing the certainty of the first order moment estimation. This is based on 
the assumption that the OFDM timing is approximately aligned due to the fine tuning of the packet synchronization $\zeta^{\prime}$. By definition, the cross correlation between a pair of energy signals, $x[n]$ and $y[n]$, is given by [24]

$$
r_{x y}=\sum_{n=-\infty}^{\infty} x[n] y[n-\chi], \quad \chi=0, \pm 1, \pm 2, \cdots,
$$

where the parameter $\chi$ is called lag and it indicates the time-shift between the pair. Based on this theory, the time-shift in samples for either expansion or compression can be measured with respect to a reference sequence length of the guard interval $N_{g}$. In the case of the existence of Doppler shift, the received samples are shifted to the right in expansion or left in compression with respect to the reference. To be more specific, once the start of the packet $\zeta^{\prime}$ is identified, it can be deemed that the symbol timing identification is reliable and the correlation between the received $\mathrm{CP}$ and its replica is computed to measure the time-shift in the samples.

$$
\begin{aligned}
& \Lambda_{c} \triangleq\left|\sum_{n=0}^{N_{g}-1} r\left(\zeta^{\prime}+n\right) r\left(\zeta^{\prime}+n+N-\chi\right)\right|, \\
& \chi=0, \pm 1, \pm 2, \cdots .
\end{aligned}
$$

Considering that the reference sequence of the transmitted CP is $N_{g} N_{s}$, where $N_{s}$ denotes number of samples per symbol, then the first order moment of the Doppler shift $\phi_{y y}$ can be approximated as

$$
\begin{aligned}
& \hat{\phi}_{y y}=\arg \max \Lambda_{c}-N_{g} N_{s}, \\
& \chi=0, \pm 1, \pm 2, \cdots .
\end{aligned}
$$

In this paper, instead of a single estimation of the first order moment, it has been estimated by the collaboration of centroid-based localization $\phi_{l}$, auto correlation of the cyclic prefix with its Doppler-shifted replica $\phi_{y y}$ and first order expectation $\phi_{E}$. In such a case, the conditional expectation of $\phi$ is given as

$$
\hat{\phi}=E(\phi / \tilde{\phi})=E[\phi / \tilde{\phi}(0), \phi / \tilde{\phi}(1), \phi / \tilde{\phi}(2)],
$$

where the symbol $E$ denotes the expectation operator and $\tilde{\phi}=\left[\tilde{\phi}_{l}, \tilde{\phi}_{y y}, \tilde{\phi}_{E}\right]$ is a row vector of scalar real values that contains noise within the measurements. For the first OFDM symbol $j$, the estimation of the first order moment $\hat{\phi}_{j}$ is based on averaging $\tilde{\phi}_{l}$ and $\tilde{\phi}_{y y}$. However an additional parameter is added which is based on the linear expectation $\tilde{\phi}_{E}$ as mentioned in [25], therefore the estimated mean shift in samples $\bar{\phi}$ is given as

$$
\bar{\phi}_{j}=\frac{\tilde{\phi}_{l}+\tilde{\phi}_{y y}+\tilde{\phi}_{E}}{3}, \quad \text { for } j>2
$$

1) Control range and PM algorithms: The parameter $\tilde{\phi}_{E}$ can only be considered reliable with increasing or decreasing gradient, i.e., when the speed change is unidirectional during a packet time. However, this is an unrealistic condition, where the speed could be steepening and leveling off during the packet time. Therefore, it is crucial to govern the estimation within a specific range to detect anomalous situations. This range is the first part of the PM and it is built on the assumption that the speed is increasing with the packet time at constant acceleration. 
Based on that, the system is capable of predicting the drift in samples in the next symbol. Let us define a new variable $\phi_{a}$ to buffer the absolute difference between $\hat{\phi}_{c}$ and $\hat{\phi}_{p}$

$$
\phi_{a}=\left|\hat{\phi}_{c}-\hat{\phi}_{p}\right|
$$

where $\hat{\phi}_{c}$ and $\hat{\phi}_{p}$ represent the current and previous estimation at time $j$ and $j-1$ respectively determining the mean value $\bar{\phi}_{a}$ of (23) over the OFDM blocks. Accordingly, we formulate a general expected range in samples $\hat{\phi}_{C}$ and it can be written as

$$
\hat{\phi}_{C} \leftarrow \hat{\phi}_{j-1} \pm 2\left|\bar{\phi}_{a}\right|
$$

where the (+) sign indicates an acceleration in the expansion of the signal since the distance is increased and vice versa. An algorithm is developed in (1) to deal with these scenarios.

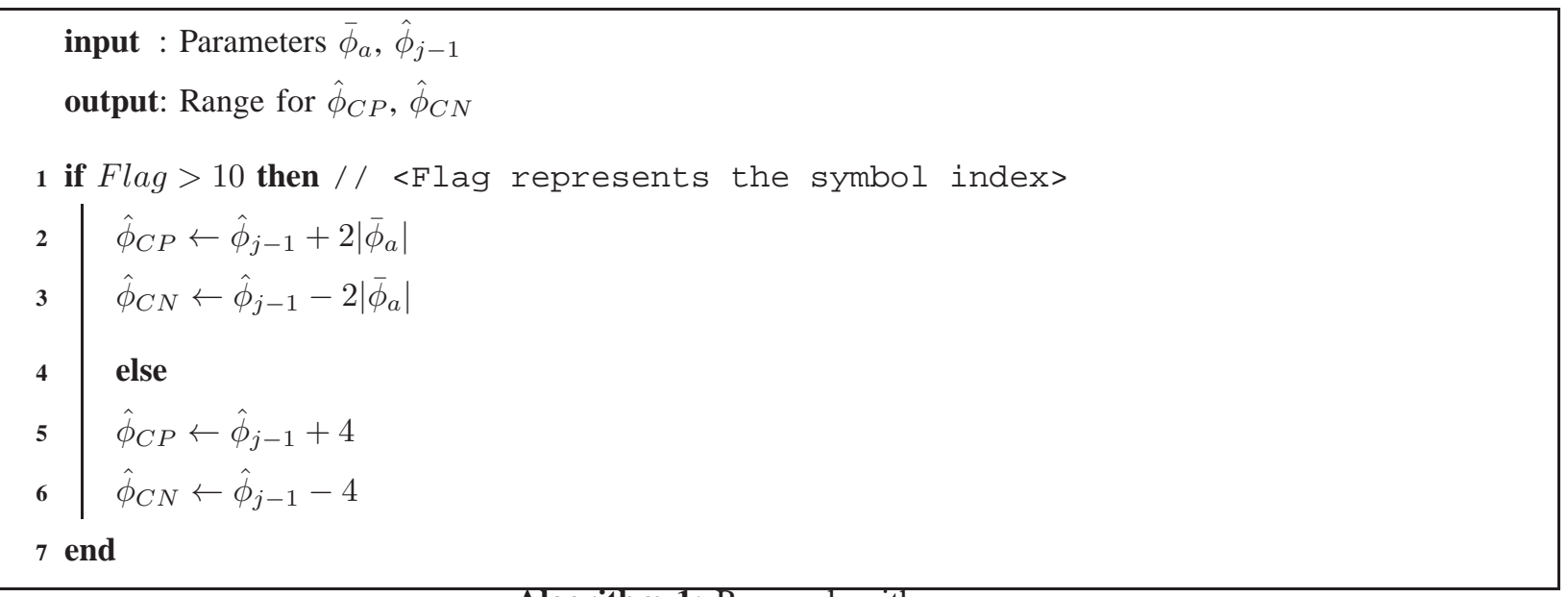

Algorithm 1: Range algorithm

It can be noticed from algorithm (1) that $\hat{\phi}_{C P}$ and $\hat{\phi}_{N P}$ ranges are assigned for the positive and negative acceleration, respectively. For instance, if $\hat{\phi}_{j-1}=5$ samples and the average drift in samples of the the previous 10 OFDM blocks were $\bar{\phi}_{a}=2$ samples, then, it is expected that $\hat{\phi}$ will be $\hat{\phi}_{i-1} \mp 2$. Accordingly, in algorithm (1), lines 2 and 3, we expand the range to a square half of this coefficient. In this case the range is expressed as $\left[\hat{\phi}_{j-1}-2\left|\bar{\phi}_{a}\right|, \hat{\phi}_{j-1}+2\left|\bar{\phi}_{a}\right|\right]$ instead of $\left[\hat{\phi}_{j-1}-\left|\bar{\phi}_{a}\right|, \hat{\phi}_{j-1}+\left|\bar{\phi}_{a}\right|\right]$. In lines 5 and 6 on the other hand, $\hat{\phi}_{j-1} \mp 4$ is based on the assumption that $a=1 \mathrm{~m} / \mathrm{s}^{2}$. In this case the speed will change $\mp 0.25 \mathrm{~m} / \mathrm{s}$ in each OFDM symbol and this can be interpreted in terms of samples to $\mp 2$ samples. As in lines 2 and 3, the tolerance is also increased by 2 .

The second step in the PM is to set the conditions that are needed to make an action to correct the estimation. There are three cases adopted here to perform the PM. In each case, two out of three parameters are considered and the third one is dropped. This procedure is necessary in order to accommodate the abrupt change in the direction of the velocity, hence the range control detects this perturbation in the speed while the PM applies the appropriate action by ignoring the nuisance parameter. Consequently, the average of the reliable parameters are considered and utilized in the search. This procedure of PM is shown in algorithm (2) 


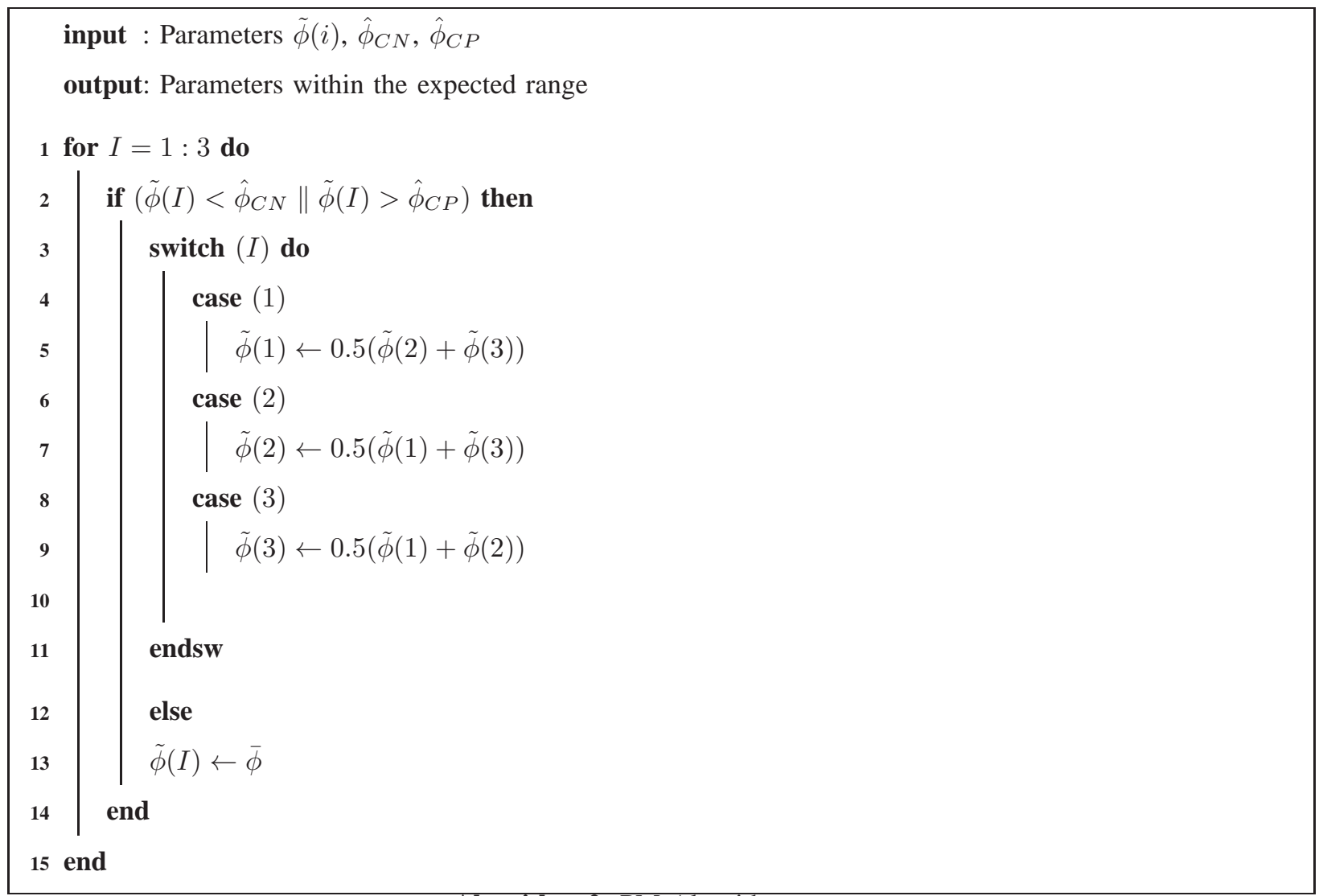

Algorithm 2: PM Algorithm

\section{Early termination search algorithm}

In this algorithm, we are trying to estimate and compensate the time varying Doppler shift recursively. An adaptive step-size is formulated in accordance with a number of iterations to obtain an optimal search that results in a minimum number of errors. The criteria of optimality is adopted here in the sense of performance investigation, therefore, the CRC is employed to terminate the search swiftly once there is zero decoding errors. On the other hand, this search algorithm reveals the minimum phase error and their accompanied parameters that give the lowest BER to be utilized later in the outer iteration. This outer iteration is enabled when the search algorithm fails to produce zero decoding errors.

1) Selection of step-size $(\mu)$ and correction factor $\left(K_{i}\right)$ : For a closed-form system that contains several instantaneous variables, the estimation of the required parameter is generally not possible [26]. An alternative solution to approximate the parameter is adopting an iterative approach. The estimation of the parameter at iteration $I$ represents the initial expectation and then this estimation is resumed recursively to improve it. Based on this approach, the parameter $\hat{\phi}_{I-1}$ which was fine tuned earlier to produce the minimum number of errors among three estimation agents, is utilized. The adaptation factor is shown as

$$
\mu=0.33\left(\frac{\operatorname{sgn}(I / 2)\lfloor|I / 2|\rfloor}{0.5 \ell}\right)^{n},
$$




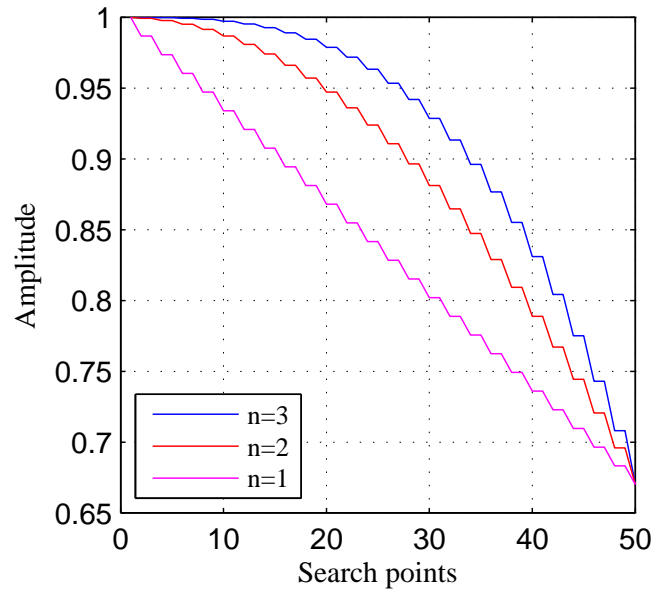

(a) Change of correction factor as a function of the exponents.

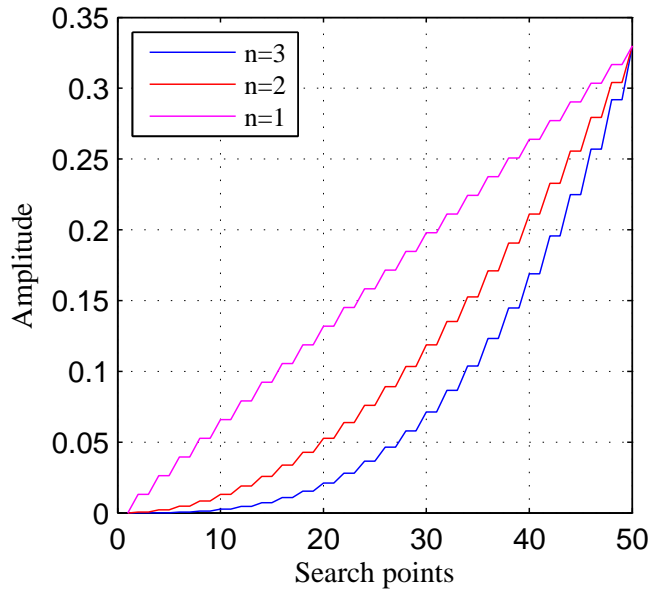

(b) Change of step-size as a function of the exponents.

Fig. 4. Effect of exponents on step-size and correction factor convergence.

where $n$ is a positive integer exponent and $\ell$ represents the search points. The search is chosen to converge the correction term given in (26) towards minimizing the phase error and reliable estimation of $\hat{\phi}$. This correction term is initialized to 1 and then approximated iteratively. The idea behind selecting a cubic exponent step size is to search in a convergent manner as the cardinality of estimation is high at the beginning and once it diverges the estimation error is expected to be increased. In terms of complexity, this search algorithm is better than a linear approach, where it requires higher execution time. In addition, this type of search has an automatic early termination (AET) condition. This termination depends on:

1) The CRC results 0 errors,

2) Reaching the maximum search points.

Resorting to the iteration is to practice another step-size and correction term that should be selected closer to those at previous iteration. In this manner, it is devised that the search algorithm diverges one step per iteration around the range $[\ell / 2]$ towards left and $[\ell / 2]$ towards right. An action is taken in case of reaching the full range by considering the estimated $\hat{\phi}$ at iteration $i$.

$$
K_{i}= \begin{cases}\mu+1 & \bmod (i, 2)=0 \\ \mu-1 & \text { otherwise }\end{cases}
$$

As shown in Fig. 26, the exponent $n$ of the step-size in (25) plays an important role in reducing the errors. Although a higher degree of exponent indicates that the estimation is reliable, there is a level at which no improvement gain is obtained.

It is shown in Fig. 4(b) that at $n=3$, the horizontal asymptote starts smoothly during the first 10 candidate points of the search range, whereas the smoothness period is smaller when $n=2$. On the other hand, in a linear case $n=1$, the step-size is constant. 
The implications of the step-size in (25) are shown in Fig. 4(a). In this figure, the correction factor $K_{i}$ is changing in accordance with the step size to ultimately force $\hat{\phi}_{c}$ to converge. However, failing to attain an improvement and ultimately converging to AET condition 1 results in an increased estimation error, hence the correction term in (26) diverges and then the search algorithm starts to choose a larger step-size.

2) Time varying Doppler shift estimation and tracking: In terms of performance, when $n=3$, it can be inferred that the reliability of estimating $\hat{\phi}_{c}$ in (18) is high and is only needed to fine tune the approximation, thus it necessitates adjusting the step size closer towards the left or right around the middle of the search range. In this case fine tuned $\hat{\phi}_{c}$ is obtained. It represents the timing offset at the start of the OFDM symbol, which is approximated as

$$
\hat{\phi}_{c}=\tilde{\phi} K_{i}
$$

The parameter $\hat{\phi}_{c}$ contributes to the improvement of other dependent parameters, particularly, the tracking step. Therefore, the iterative approach represented by the search is important to approximate the Doppler shift estimation. To be more specific, let us assume that the speed between the transmitter and receiver is $1 \mathrm{~m} / \mathrm{s}$, which is equivalent to $8 \mathrm{~Hz}$ for a carrier frequency of $12 \mathrm{kHz}$ and sampling frequency $4 f_{c}$, therefore, $\hat{\Delta}=1.0006$ and the estimated $\phi$ should be 2.048 for 12288 FFT up-sampling. Actually, these calculations yield that there is a demand on estimating and compensating such Doppler shift that has a fraction of a variable time expansion and/or compression. Therefore, dealing with such time varying Doppler shift necessitates tracking this variation within the symbol time. In [25], this Doppler is dealt with by deriving a tracking step to estimate this variation based on dividing the time varying Doppler shift into an integer part for re-sampling and its residual or CFO is represented by the fractional part of the Doppler shift and smoother Doppler shift estimation is obtained. However, in the proposed adaptive system, recursive iteration to fine tune $\hat{\phi}_{c}$ is adopted and the time-varying Doppler shift contains the re-sampling factor with its residual. Furthermore, it has been dealt with CFO estimation separately, hence the Doppler shift is given as

$$
\hat{\Delta}(k)=\frac{L_{f}-\hat{\Psi}(k)}{L_{f}}
$$

where $\Psi(k)$ is the sampling frequency offset initialized with $\hat{\phi}_{s}$ at $k=1$ and its update is approximated as

$$
\Psi(k)=\Psi(k-1)+\hat{\phi}_{\text {step }},
$$

where $\hat{\phi}_{\text {step }}$ is given as

$$
\hat{\phi}_{\text {step }}=\frac{\hat{\phi}_{e}-\hat{\phi}_{s}}{L_{f}}
$$

Utilizing the $4 t h$ order Lagrange interpolation polynomial [27] for re-sampling based upon the parameter $\hat{\Delta}(k)$ to produce $r^{\prime}(k)$, this re-sampled signal can be mathematically written as 


$$
r^{\prime}(k)=\sum_{i=0}^{N} \tilde{r}_{m^{\prime}}[k(1+\Delta(k))] V_{i}\left(m^{\prime}\right),
$$

where

$$
V_{i}\left(m^{\prime}\right)=\prod_{i=0, n \neq i}^{N} \frac{m^{\prime}-m_{n}^{\prime}}{m_{i}^{\prime}-m_{n}^{\prime}}
$$

where $V_{i}\left(m^{\prime}\right)$ represents the polynomial of degree $N$ associated with each node $i$ where $m^{\prime}=m^{\prime}+\hat{\Delta}(k)$ initiated with $3, n \in\{1,2, \ldots N\}, R=\left\lfloor m^{\prime}\right\rfloor$ and $m_{i}^{\prime}=R_{i-2}$. Therefore, for five points $N=4$, the current point, the two previous points and next two points are considered to fit the interpolation curve.

3) Residual Doppler shift estimation : Post-FFT CFO estimation is adopted. When all angles of the received $n t h$ pilots $Y_{p}(n)$ are shifted by the same angle, the ZFE is capable of correcting the rotation. However, this is not the case where each sub-carrier is rotated depending on the residual Doppler-shift. In order to estimate this residual, a range of these parameters are assumed. Start with $\hat{\epsilon}$ and for each candidate $i$, the phase error vector is determined as

$$
\hat{e}_{i}(n)=\sum_{n=0}^{N_{p}-1} \angle\left(Y_{p}(n)\right)-\angle\left(X_{p}(n)\right),
$$

considering the mean phase error between the transmitted and received pilots that is given as

$$
\bar{e}_{i}=\frac{\sum_{n=0}^{N_{p}-1} \hat{e}_{i}(n)}{N_{p}}
$$

therefore, the estimated residual phase error at the pilots sub-carriers indices can be formulated as

$$
\hat{e}_{r}=\sum_{n=0}^{N_{p}-1}\left|\hat{e}_{i}(n)-\bar{e}_{i}\right|,
$$

and the CFO can be approximated as a function of this pilot-based residual phase error estimation

$$
\hat{\epsilon}^{\prime}=\epsilon \underset{\min }{\left(\hat{e}_{r}\right)}
$$

This criteria denotes the CFO candidate that accompanies the lowest phase error. Hence, after re-sampling, the resulting received signal $r^{\prime}(t)$ is then down-converted to baseband with the chosen $\hat{\epsilon}^{\prime}$ and can be written as

$$
r(t)=r^{\prime}(t) e^{-j 2 \pi\left(f_{c}+\hat{\epsilon}^{\prime}\right) k T_{s}} .
$$

After compensating the CFO in (37), the resulting signal $r(t)$ is converted to the frequency domain and delivered to the ZFE. To improve the receiver performance, post-FFT tracking is useful to mitigate the remaining CFO [22]. Although this residual is small, it degrades the receiver performance due to the accumulated phase rotation consequences along each OFDM symbol in the packet [28]. On the assumption that the CFO is compensated earlier, 


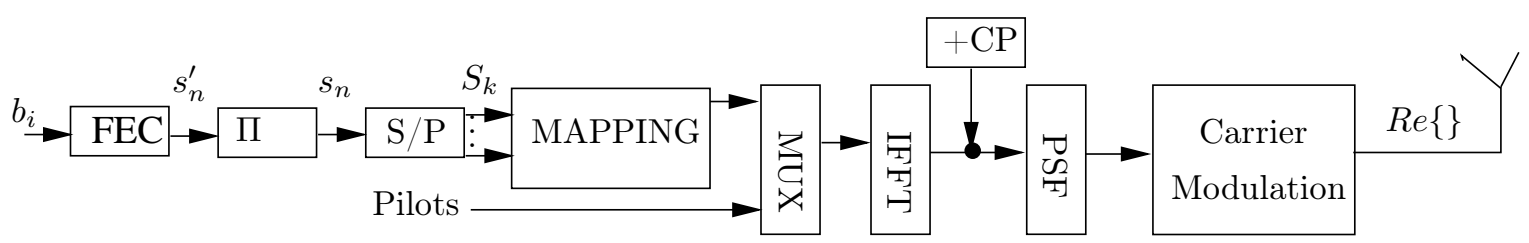

Fig. 5. Structure of the transmitter used in simulation.

it is worth eliminating its phase rotation effect. Here in this proposed technique, we utilize the pilots to estimate the residual phase error of the current OFDM symbol which can be written as

$$
\theta_{i}=\angle(Y(n))-\bar{e}_{i}
$$

and the residual phase error correction is written as

$$
\hat{r}=Y(n)=e^{j \theta_{i}}
$$

which is the OFDM signal after residual phase correction. This yields to deliver reliable information to the BICM-ID decoder.

Based on the CRC, a decision is made to terminate the search or resume the iteration. In Fig. 2, it can be seen that once the search points are completed and there is an error after performing the CRC, which is assigned a dashed line, the outer iteration is enabled as a final trial. To deal with this case, the phase error given in (33) is utilized to buffer the associated parameters $\hat{\phi}_{c}^{\prime}$ and $\hat{\epsilon}^{\prime \prime}$ that result in minimum phase error during previous iterations. Exploiting $\hat{\phi}_{c}^{\prime}$ updates the Doppler shift and produces a new interpolation factor whereas $\hat{\epsilon}^{\prime \prime}$ is utilized in compensating the CFO. In order to distinguish the CFO at each stage, we use the variable $\hat{\epsilon}^{\prime \prime \prime}$, which denotes the output of the multiplexer among three estimations.

\section{System DESIGN, SIMULATION, AND EXPERIMENTAL RESUltS}

Fig. 5 depicts the structure of the transmitter used in simulation. For the sake of simplicity, it is assumed that the system under consideration does not account for the PAPR, as in the experiment. As mentioned in (II), the binary information bits $b_{i}$ are applied to the FEC to produce a codeword, $s_{n}^{\prime}$. The interleaved bit sequence $s_{n}$, is then converted in groups of $m$ successive bits into alphabet symbols of constellation size $M=2^{m}$. After this mapping operation, the OFDM frame which contains CP is constructed. The resulting frame is pulse shaped and then up-converted using carrier modulation.

The transmitted signal $x_{i}(t)$ in (3) is passed through the channel model shown in Fig. 6. This model is adopted to imitate the case of the time varying Doppler-shift with constant acceleration. Performing this type of simulation necessitates designing a packet structure which contains multiple OFDM symbols to accommodate the required acceleration. As mentioned earlier, the LFM signal is utilized for packet synchronization, however, the effect of acceleration on the chirp is not negligible with such a type of packet transmission. Therefore, the chirp also undergoes 


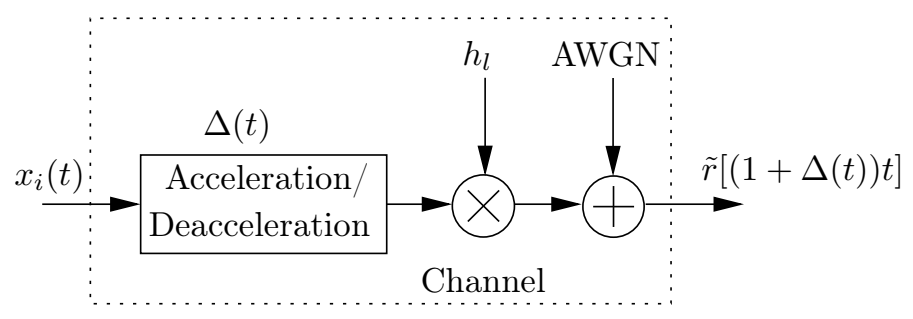

Fig. 6. Simulation model.

this effect in the simulation, hence it is involved in the acceleration and deceleration of the first and second packet, respectively. It should be stressed that there is an acceleration in the expansion case or in the compression, similarly for deceleration. This is illustrated in Fig. 7 where the uphill and downhill of the solid line mean there is a change in the direction of the velocity with time, i.e. inflection point from acceleration to deceleration, whereas the flat line means that the relative velocity between the transmitter and receiver is constant or zero acceleration over the duration of the packet. Likewise for the dotted line in this case, the velocity increases towards the negative in the first packet then starts decreasing towards the positive in the second packet. Accordingly, the simulation uses two consecutive packets to imitate the proposed system, namely packet 1 and packet 2 . The first was accelerated and the second was decelerated.

In simulating the time varying Doppler shift, the speed was assumed initially equal to zero and then the terminal speed of the packet is given as

$$
\begin{aligned}
V_{\max }(t) & =\sum_{a=0}^{a_{\max }} \frac{a(t) L_{\mathrm{pac}}}{f_{s}} \\
& =\sum_{a=0}^{a_{\max }} a(t) \cdot T_{\mathrm{pac}}
\end{aligned}
$$

where, $a(t), a_{\max }$ represent the acceleration and its maximum, respectively, $L_{\mathrm{pac}}$ is the total samples of the packet, $f_{s}$ is the sampling rate and $T_{\text {pac }}$ is the packet duration. Therefore, the associated Doppler shift at the end of the packet relative to the propagation speed can be written as

$$
\Delta_{\max }(t)=\frac{c-V_{\max }(t)}{c},
$$

where $\Delta_{\max }(t)$ represents the Doppler shift at $t=T_{\text {pac }}$. Based on the assumption that the speed is changing linearly during the packet duration, then at each sample time within the OFDM symbol, the first order Doppler shift is formulated as

$$
\Delta_{\text {step }}=\frac{\Delta_{\max }(t)-\Delta_{0}}{L_{\mathrm{pac}}}
$$




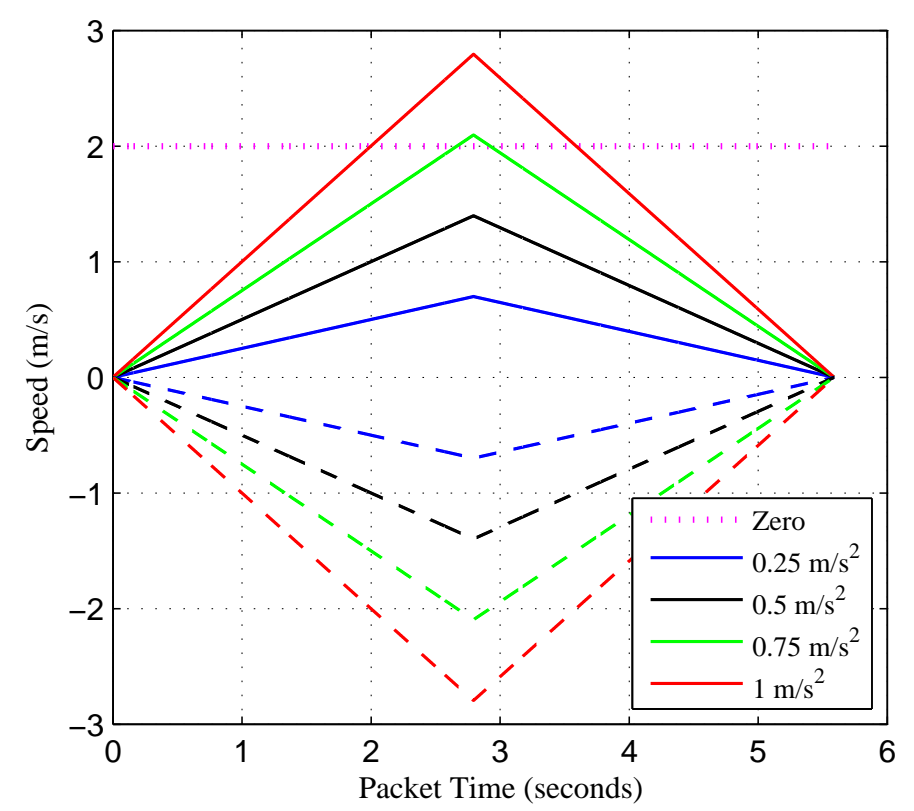

Fig. 7. Scenarios of time-varying Doppler shift.

where $t=T_{\text {pac }}$. Based on this step, the Doppler shift is speeds up until arriving at the last symbol in the first packet and then starts slowing down. It is well known that there are lots of UAC channels that have been characterized, yet there are no standards as in the case for RF channels [29]. Therefore, the channel model in [30] is adopted and the subsequent stage is to convolve the time dispersion channel with the Doppler shifted incoming signal, prior to adding the AWGN for investigating a more realistic case.

\section{A. System design parameters}

1) Transmitted packet structure: The packet structure used in the simulation is shown in Fig. 8. The system bandwidth of $8 \mathrm{kHz}(8 \mathrm{kHz}-16 \mathrm{kHz})$ is swept by a $50 \mathrm{~ms}$ chirp which prefixes a packet. The signal packet comprises 10 CP-OFDM frames of QPSK. The length of each QPSK OFDM frame plays an important role in controlling the performance of the Doppler shift estimation and compensation. In addition, required Doppler resolution and acceleration contribute in determining the OFDM frame and packet length, respectively.

2) Parameters of cyclic prefix: Due to the symmetry of the $\mathrm{CP}$ with its replica, there is a good correlation property of this guard interval denoted as cyclostationary because there is a cyclic convolution with the channel in the time domain. However, depending on the transmitted data, resulting envelopes of the correlation peaks and their sidelobes are varied. Particularly, if the transmitted data are random, the peaks and sidelobes are variable whereas with symmetrical data (the start and end of the frame contain the same data) the peak-to-average power ratio is symmetrical. Since the Doppler is changing with time, there is a mismatch in the CP correlation, where the Doppler affects the first part of the CP by a different amount to the second CP part. This mismatch appears in the position 


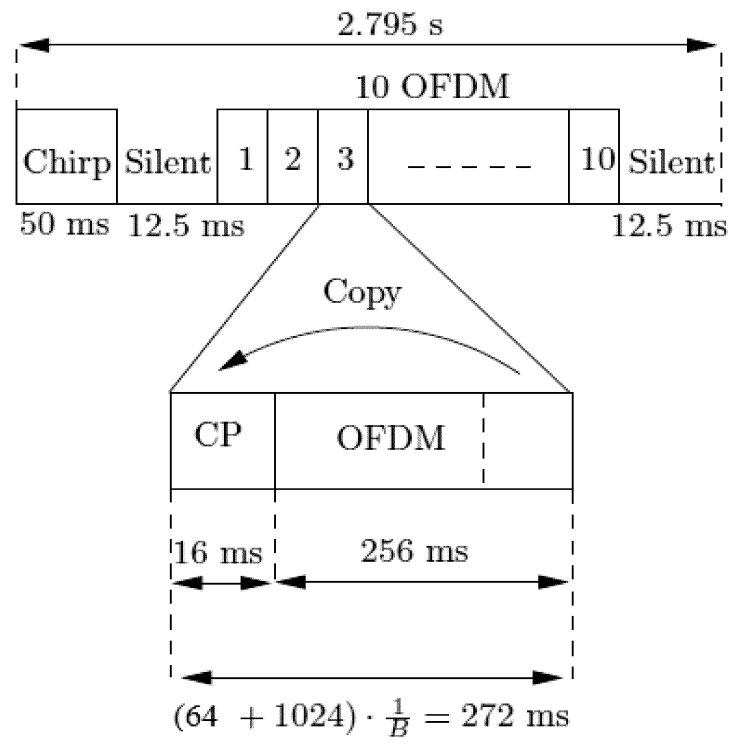

Fig. 8. Packet structure for $N_{c}=1024$.

of the $\mathrm{CP}$ and this case is dealt with by searching within a specific window around the leading and trailing edge of the OFDM symbol. Furthermore, there is a mismatch in the length of the CP windows. To be more specific, let us assume the acceleration is $1 \mathrm{~m} / s^{2}$ and the Doppler frequency shift at CP1 is $F_{d}$, then we expect $F_{d} \pm 2 \mathrm{~Hz}$ at $\mathrm{CP} 2$ when the symbol time is $0.25 \mathrm{~s}$. This frequency shift is ignored relative to $f_{s}$ and $N_{g} \cdot N_{s}$. Therefore, reducing the $\mathrm{CP}$ length could be useful in terms of its sensitivity to the Doppler shift and bandwidth.

The bandwidth of the cyclic prefix is chosen to accommodate the channel impairments and to minimize the loss of data rate. In this case, the coherence bandwidth lower bound is given as

$$
B_{c}(\text { lower })=\frac{N_{c}}{N_{g}} \Delta_{f} .
$$

Furthermore, the BT product is subject to the required amount of gain to achieve reliable detection. A gain of (18 $\mathrm{dB})(\mathrm{BT}=64)$ was determined in accordance with the OFDM signal design to be sufficient, and therefore, the cyclic prefix is a $16 \mathrm{~ms}$ period.

\section{B. Simulation results}

In this section, we present the simulation results of the proposed system based on the simulation model described in Fig. 6. The CIR was $h(n)=0.6708 \delta(n)+0.5 \delta(n-1)+0.3873 \delta(n-2)+0.3162 \delta(n-3)+0.2236 \delta(n-4)$. In order to investigate the system performance, two scenarios are considered: acceleration (expansion) and deceleration (compression) up to $1.1 \mathrm{~m} / \mathrm{s}^{2}$. Fig. 9 shows the output of the centroid-based correlation. It can be seen in this figure that the length of the correlation window in the $\mathrm{x}$-axis is 100 samples and the centre is 50 as in the case of zero 


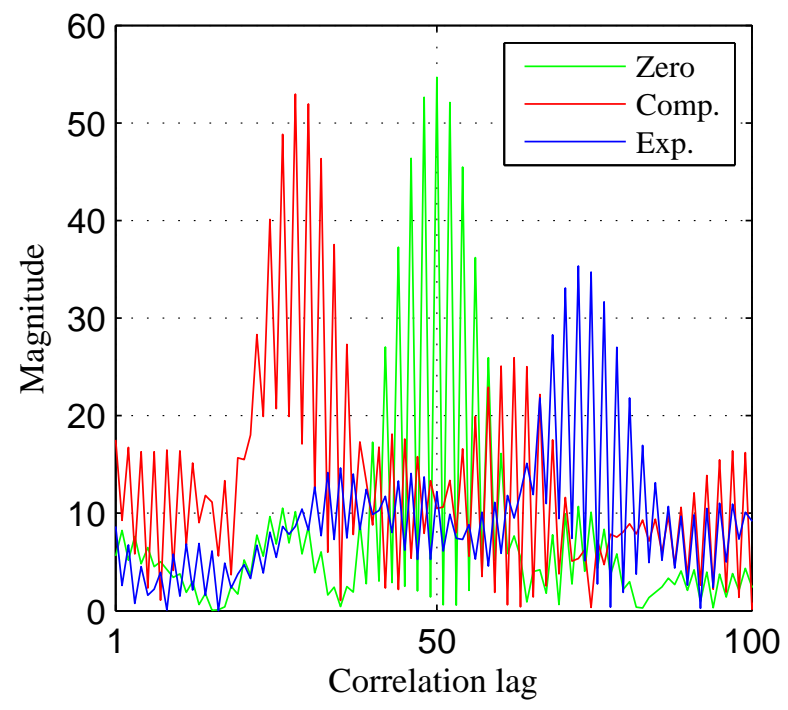

Fig. 9. Correlation lag variation due to time scale expansion/compression at an acceleration of $1 \mathrm{~m} / \mathrm{s}^{2}$.

Doppler shift, therefore any drift relative to this centre due to an expansion or compression is exploited to estimate the timing offset. Fig. 10 shows a plot of the BERs at $\mathrm{SNR}=15 \mathrm{~dB}$ and a maximum delay spread of $10 \mathrm{~ms}$ for $N_{c}=1024$. In order to assess the proposed system with the two scenarios, different accelerations and various $\mathrm{CP}$ lengths were used. For $\mathrm{CP}=32$ or $8 \mathrm{~ms}$, the system fails in all scenarios and at different accelerations. This is due to the severe ISI that introduces a delay spread greater than the CP length. However, for $\mathrm{CP}=64$ and 128, the receiver achieves a satisfactory performance through all anticipated accelerations and scenarios. A clear benefit of increasing the $\mathrm{CP}$ length over shorter $\mathrm{CP}$ is in the low acceleration case. This is palpable in Fig. 10(a) at $a=0.3$ $\mathrm{m} / \mathrm{s}^{2}$. This means, at low accelerations we need to increase the resolution of the estimation by extending the CP length. In contrast, at higher accelerations, the impact of increasing the CP length on the performance is marginal as shown in Fig. 10(b).

Fig. 11 shows the performance of the time varying Doppler-shift compensation versus the delay spread of the channel. There was a significant reduction in the BER over a short delay spread. A possible explanation for this might be that shorter delay spread increases the certainty of the $\mathrm{CP}$ correlation peaks due to the increased area of the ISI free region, hence an accurate Doppler-shift is obtained. Accordingly, these simulation results confirm a trade-off in the design of an OFDM frame structure between the spectral efficiency, the desired acceleration and ICI reduction. This means the guard time $T_{g}$ should not only be chosen to achieve the condition $\geq \tau_{\text {max }}$, rather it should also consider what is the required maximum velocity that attain the optimal performance. Therefore, with the design parameters of $T_{g}=16 \mathrm{~ms}$ and $N_{c}=1024$, it is verified from these simulation results that the system can attain a BER of $10^{-5}$ at $a= \pm 0.5 \mathrm{~m} / \mathrm{s}^{2}$ and $\tau_{\max }=5 \mathrm{~ms}$.

Fig. 12 depicts alternative scenarios when a higher acceleration is simulated. In order to investigate the system 


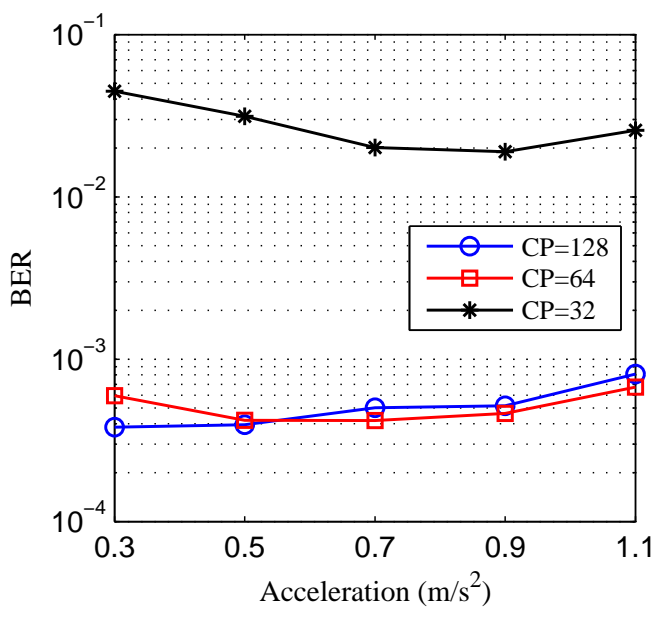

(a) Acceleration.

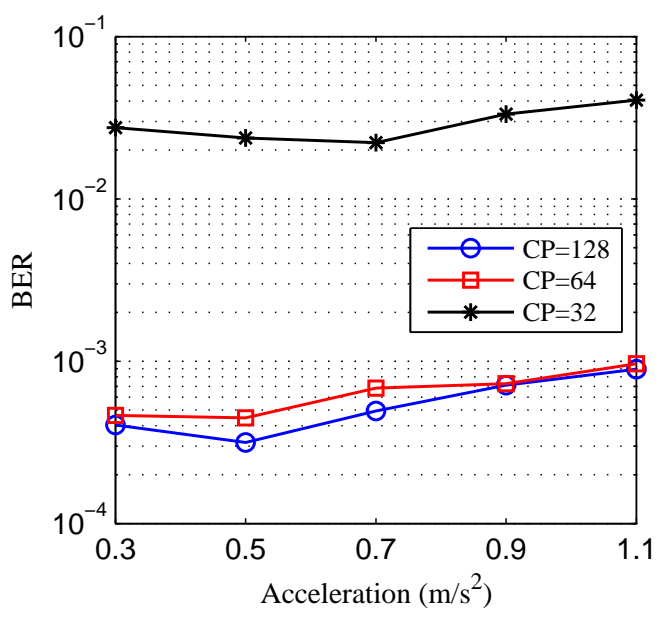

(b) Deacceleration.

Fig. 10. BERs performance for different acceleration and cyclic prefix lengths at SNR=15 dB, $\tau_{\max }=10 \mathrm{~ms}$, and $N_{c}=1024$.

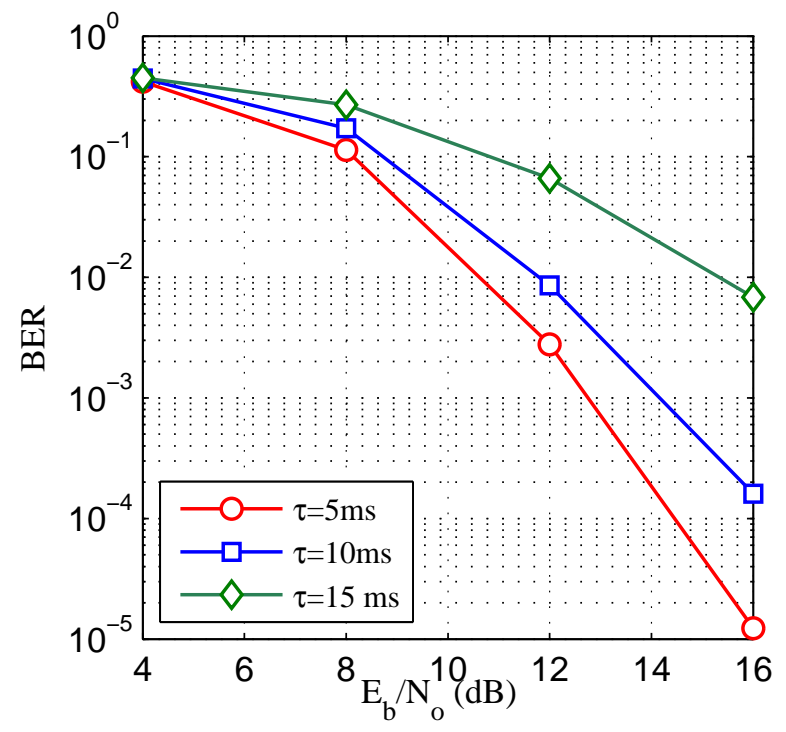

Fig. 11. Effect of the maximum delay spread $\tau_{\max }$ on the BER performance at $a=0.5 \mathrm{~m} / \mathrm{s}^{2}$ and $T_{g}=16 \mathrm{~ms}$.

performance at higher acceleration, the simulation results are firstly obtained with AWGN at $a= \pm 1 \mathrm{~m} / \mathrm{s}^{2}$ and then under the influence of the multipath channel. It can be shown that the performance of the proposed system in a combination of broadband time varying Doppler-shift and multipath channels can achieve an acceptable performance. These results suggest that the proposed adaptive receiver can accommodate a multipath channel of $\tau_{\max }=10$ ms and time varying Doppler-shift of $a= \pm 1 \mathrm{~m} / \mathrm{s}^{2}$ in the case of expansion and compression with an acceptable BER. The maximum speed that associates this acceleration at the terminal (OFDM symbol number 10) of 272 ms packet duration is $\pm 2.72 \mathrm{~m} / \mathrm{s}$. 


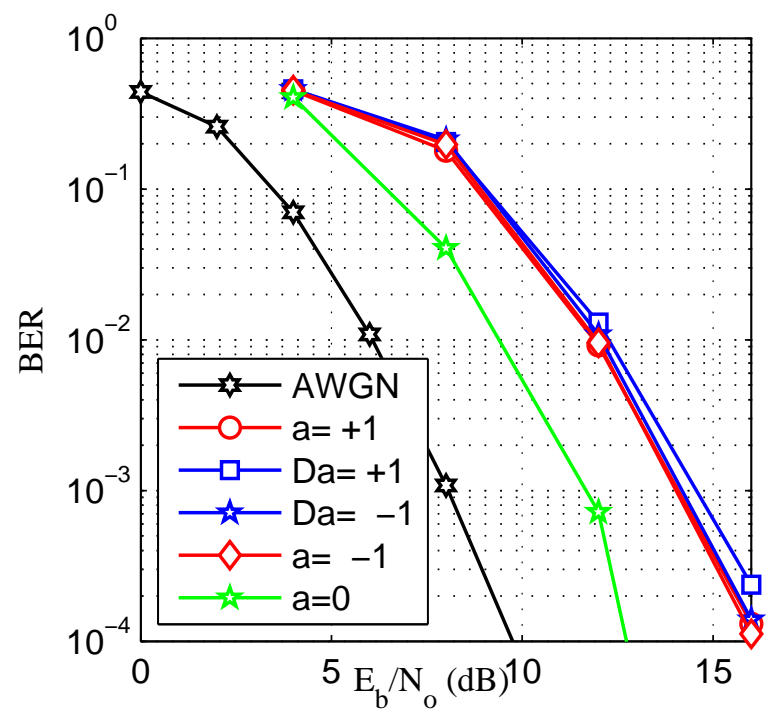

Fig. 12. BER performance with the system parameters $T_{g}=16 \mathrm{~ms}, N_{c}=1024$ at $\tau_{\max }=10 \mathrm{~ms}$ and $a= \pm 1 \mathrm{~m} / \mathrm{s}^{2}$, where Da denotes deceleration case.

TABLE I

MAIN SYSTEM SPECIFICATIONS

\begin{tabular}{|c||c|}
\hline Parameter & Value \\
\hline System bandwidth & $4 \mathrm{kHz}$ \\
\hline Carrier frequency & $12 \mathrm{kHz}$ \\
\hline Sampling frequency & $48 \mathrm{kHz}$ \\
\hline Cosine roll-off factor & 0.98 \\
\hline Code rate & $1 / 2$ \\
\hline Convolutional code polynomial & {$[23,35]_{8}$} \\
\hline
\end{tabular}

\section{Experimental results}

1) Experimental setup and channel characteristics: This experiment was conducted by Newcastle University at the UK coast. The system parameters for each OFDM frame are summarized in Table I. The transmission power was set at $108 \mathrm{~dB} 1 \mu \mathrm{Pa}$. It is known that in UAC, the multipath delay spread is inversely proportional to the distance between the transmitter and receiver and the delay spread of the short range channel is usually long. Furthermore, the presence of the Doppler shift due to the transmitter/receiver pair motion increases the burden on the receiver due to the synchronization impairments' consequences. Fig. 14 and 15 show the detected channel profiles for the 1000 and $500 \mathrm{~m}$ ranges, respectively. These figures depict the time varying CIR which is normalized to unity and the CIR of a single packet which is selected randomly. The impulse response of the channels is determined 


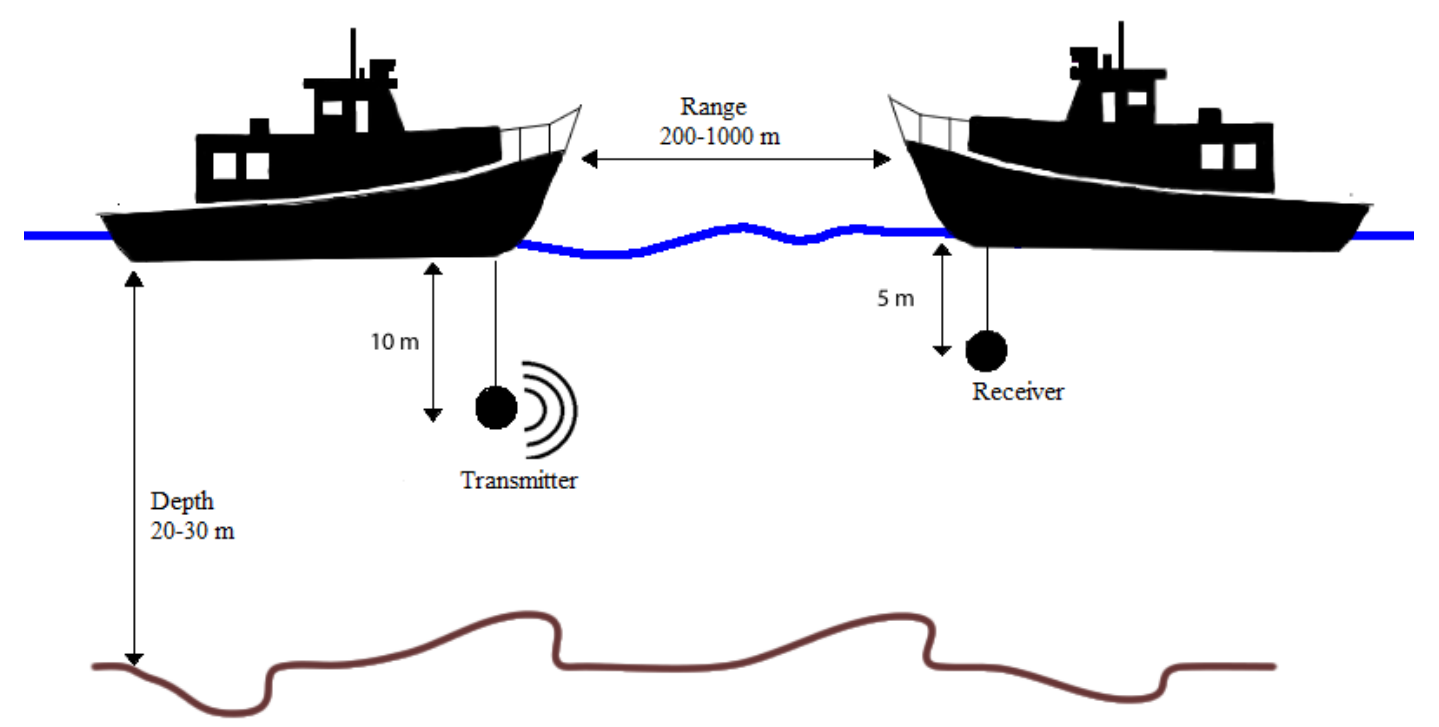

Fig. 13. Configuration of the experiment in the North Sea.

by the FIR-correlator of the LFM chirp. It can be seen that the maximum delay spread is up to 11 and $10 \mathrm{~ms}$, for 500 and $1000 \mathrm{~m}$ channel ranges respectively. However, upon a closer inspection of the CIR at the $500 \mathrm{~m}$ range, it can be inferred that it is more severe as it exhibits longer delay spread and some of their paths have a comparable amplitude relative to the direct path. Consequently, synchronization impairments challenge the receiver performance, particularly for the ZF estimator. Furthermore, in the case of $500 \mathrm{~m}$ range, it can be observed that the time difference of the arrival paths is quite significant, therefore the CIR also becomes larger [31].

2) Performance evaluation of the proposed receiver: In addition to the simulation mentioned in the preceding section, this experiment is carried out to asses the system performance. The performance of the adaptive receiver is evaluated using the packet structure shown in Fig. 8 transmitted through the multipath channels described in Section IV-C1. The chirp was used for the purpose of packet synchronization. This synchronization is achieved by correlating the LFM signal with its replica after the BPF. The highest correlation peak has been chosen to indicate the start of the packet. The performance of the proposed receiver is evaluated based on the criteria of the decoding BERs over 500 and $1000 \mathrm{~m}$ channel ranges and different OFDM data structures. CP-OFDM was used with a guard interval of $T_{g}=16 \mathrm{~ms}$ for each OFDM symbol. The number of sub-carriers used in the experiment were $N_{c}=512,1024$ and 2048 with a sub-carrier spacing $\Delta_{f}=7.81,3.906$ and $1.9531 \mathrm{~Hz}$, and OFDM symbol duration $T_{u}=1 / \Delta_{f}=0.128 \mathrm{~s}, 0.256 \mathrm{~s}$, and $0.512 \mathrm{~s}$ respectively. The code rate was $1 / 2 \mathrm{NSC}$ and a QPSK modulation scheme was utilized. Each packet comprises respectively, 20, 10 and 5 OFDM frames $N_{f}$ for $N_{c}=512$, 1024, and 2048 where the frame includes CP and OFDM symbol. The total number of information bits per packet are 8880, 


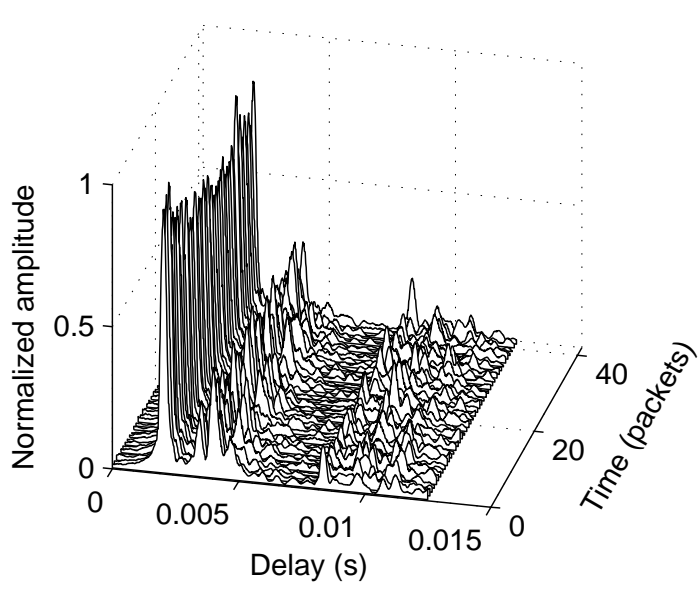

(a) Time varying CIR.

Fig. 14. Channel measurements for $1000 \mathrm{~m}$ range.

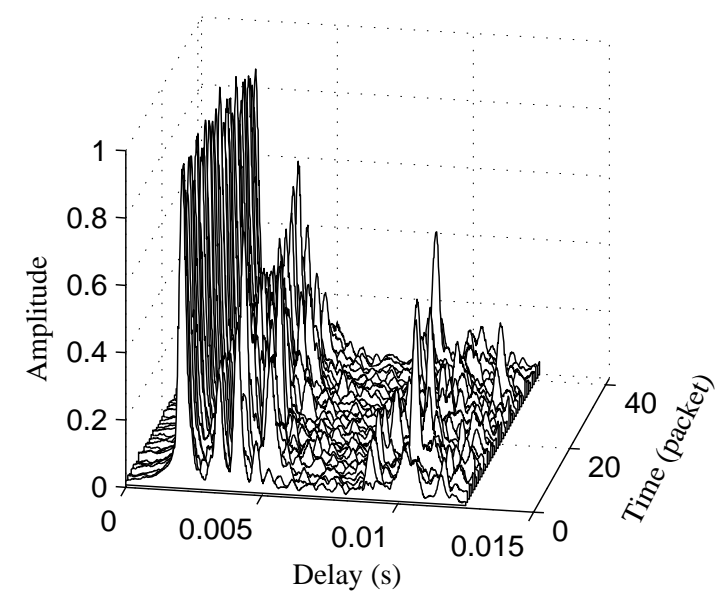

(a) Time varying CIR.

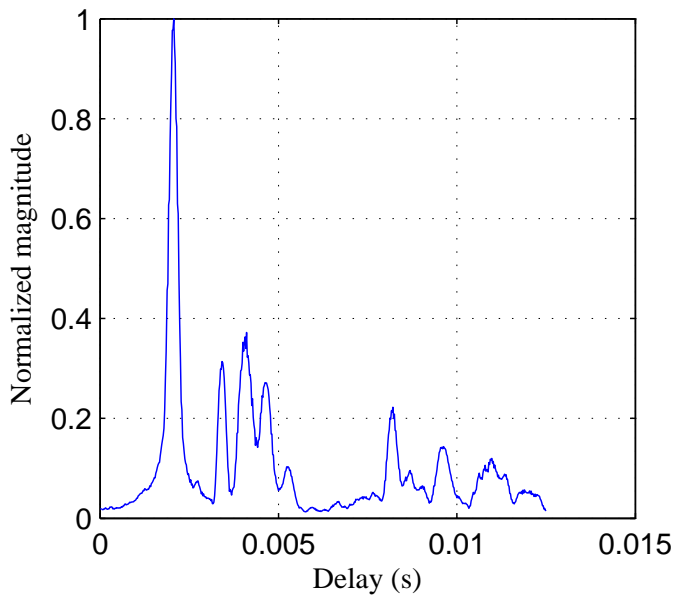

(b) Sample of CIR.

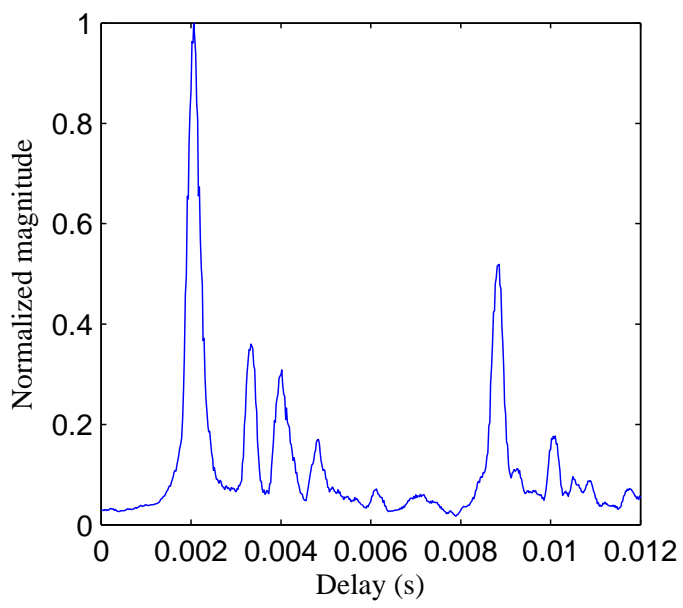

(b) Sample of CIR.

Fig. 15. Channel measurements for $500 \mathrm{~m}$ range.

8920 and 8940 for $N_{c}=512,1024$, and 2048 respectively. For $N_{c}=512,19$ packets were sent whereas 20 packets were sent for both cases of 1024 and 2048 sub-carriers. Each group of them was sent separately and at different time intervals over both channel ranges.

Fig. 16 presents the average BERs over each transmitted packet for $1000 \mathrm{~m}$ channel range and different subcarriers spacing. It can be shown from this figure that the proposed scheme surpasses the block-based Doppler-shift compensation in approximately all received packets. In total, 2 out of 20 packets have decoding errors in the case of $1000 \mathrm{~m}$ channel range and 1024 sub-carriers as shown in Fig. 16(a). The number of errors in these packets were marginal and contributed by OFDM symbol number 2 and 6 of packets 8 and 19, respectively. This result is very 
encouraging, especially with $1000 \mathrm{~m}$ range and the consequences of the associated transmission loss. On the other hand, for 512 sub-carriers, 3 out of 19 packets have decoding errors as shown in Fig. 16(b). This is an expected result, where the immunity of the OFDM sub-carriers against the ISI are proportional to the OFDM symbol length while the sensitivity to the Doppler-shift is increased with symbol time.

Fig. 16(c) presents the BER performance comparison of the proposed system technique with a block-based approach for long OFDM symbol times. It can be observed that 13 out of 20 packets are error-free. It is evident that the adaptive system is capable of achieving satisfactory performance despite its tight sub-carrier bandwidth. Additionally, this result reveals that without a CFO compensation, the receiver deteriorates as in the case of a blockbased method, therefore estimating the coarse re-sampling factor is not enough to achieve reliable communication.

As can be seen in Fig. 16(d), there are recurrent high BERs in OFDM symbol index 1 for the whole transmitted packets in the case of $1000 \mathrm{~m}$ range and $N_{c}=2048$. The underlying reason for this case is due to the false alarm in detecting the maximum peak of the chirp signal which results in synchronization impairments. Furthermore, block bit error rates (BBERs) in this figure showed that the system capable of recovering the rest of the symbols within the packet and tracking the Doppler-shift variations, where the errors are reduced dramatically with the packet time as shown in OFDM symbol 2 and 3. The reduction of the errors demonstrates that the PM is working perfectly in the process of dropping the extraordinary parameters.

Comparing the two experimental results in the case of $N_{c}=1024$ and 20484 over 500 m channel range, it can be seen in Fig. 17 (a) and (b) that despite a significant reduction in the BERs with respect to the block-based technique, the performance of $1000 \mathrm{~m}$ range surpasses $500 \mathrm{~m}$. These results are not surprising because it confirms that the associated ISI in a long distance is short due to the reduction in the delay spread length and vice versa. Through the effective contribution of the CP in estimating the Doppler-shift, this delay spread of the channel will affect the certainty of the correlation peaks, therefore room for $\mathrm{CP}$ correlation should be given. The suggestion here is to choose the $\mathrm{CP}$ longer than the channel dispersion time by at least $5 \mathrm{~ms}$ to achieve reliable communication, hence this reveals a trade-off between the bandwidth efficiency and CP length. Furthermore, longer OFDM symbol time increases the immunity against the ISI, therefore in the case of $500 \mathrm{~m}$ channel range and $N_{c}=512$, the system fails. The aforementioned results confirm that the proposed scheme achieves near error-free transmission over $1000 \mathrm{~m}$ range with a sub-carriers length of $N_{c}=1024$ or 512. In addition, although there is a narrow sub-carrier bandwidth, a satisfactory performance is achieved with $N_{c}=2048$.

3) Search points, exponents and CFO range selection: The search points, exponents selection and CFO have a direct impact on the success of the receiver operation. In the search points, reducing the complexity of the receiver is crucial, and in such a case, a limited number of inner iterations is preferred. Consequently, we resort to early termination strategy in order to reduce the computation complexity. That is, the iterations are terminated once the BER of the current OFDM symbol attains zero error on the CRC output, otherwise the receiver resumes to reach its predetermined iterations. At each iteration, two parameters are updated adaptively in accordance with the search points and the exponents. These parameters, $\mu_{i}$ and $K_{i}$ fine tune $\phi_{c}$ and an accurate Doppler-shift is obtained. Fig. 18(a) demonstrates the adaptive change of the tracking parameters $\phi_{c}, \phi_{s}$, and $\phi_{e}$ of symbol index 8 of packet 


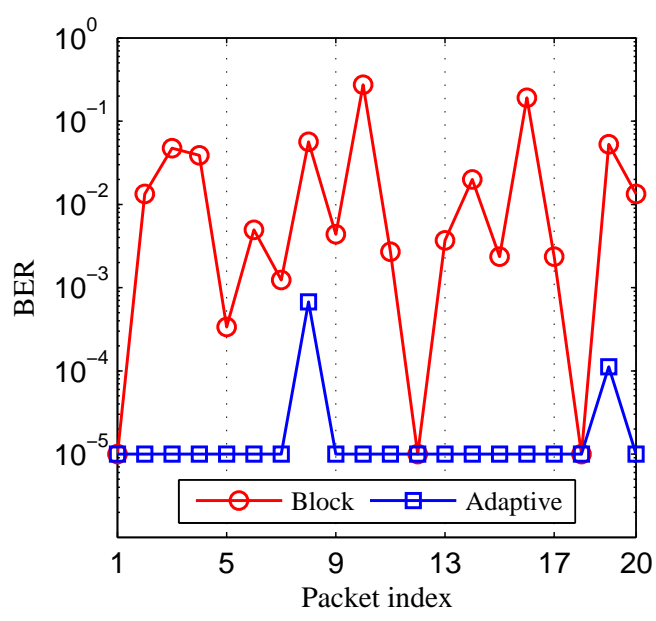

(a) $N_{c}=1024$.

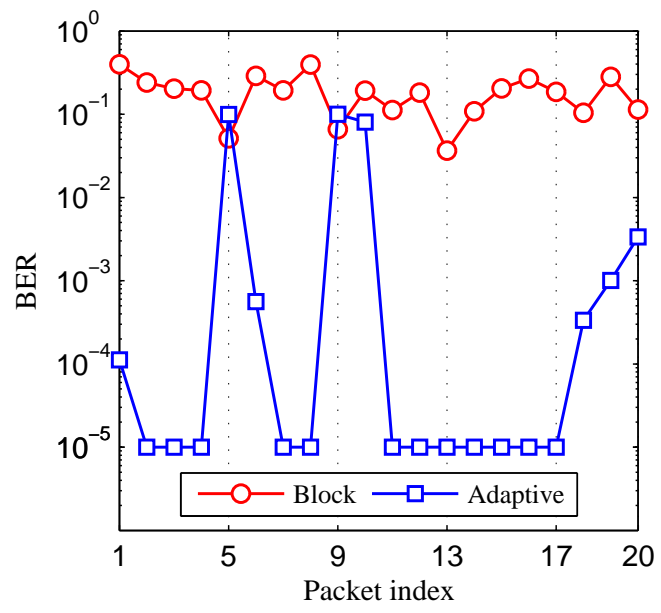

(c) $N_{c}=2048$.

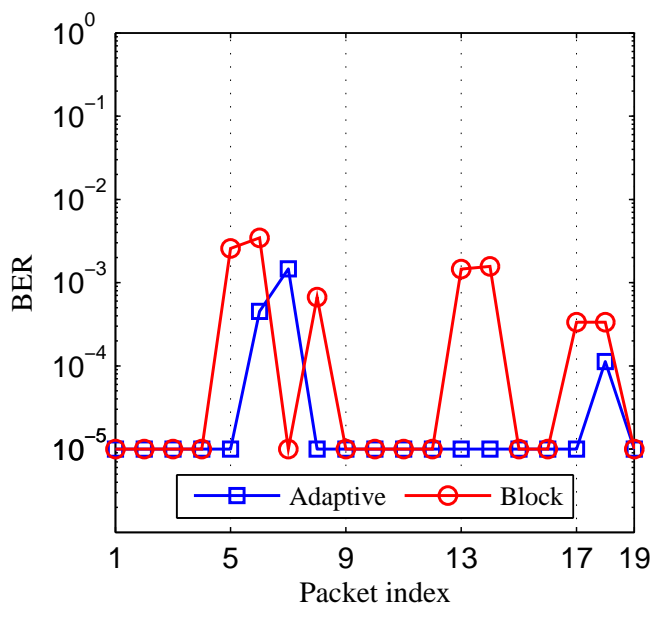

(b) $N_{c}=512$.

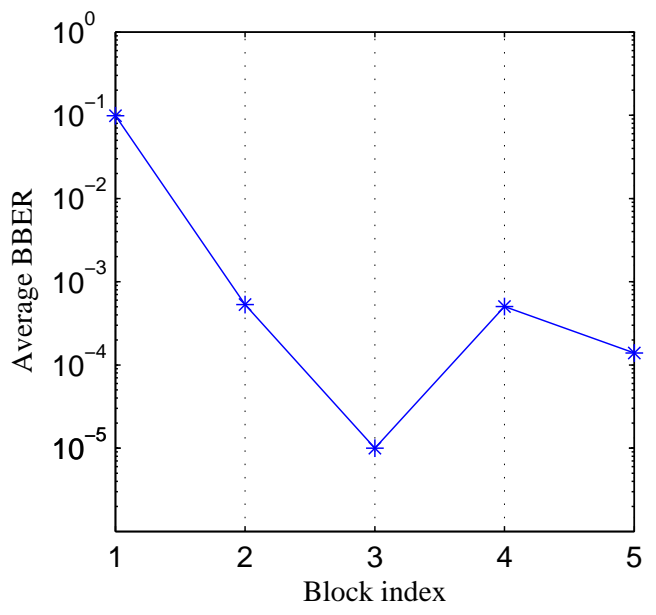

(d) Average BER per symbol $\left(N_{c}=2048\right)$.

Fig. 16. BER performance of adaptive time varying Doppler-shift compensation receiver over $1000 \mathrm{~m}$ channel range for different sub-carriers length, where the label $10^{-5}$ represents zero error.

6 with time. It is palpable from this figure that at the first iteration, the difference between them is significant which results in a large tracking step. Accordingly, the CRC test does not indicate zero error, therefore the iterations are continuing to investigate the system with further fine tuned parameters. Clearly, the step size should be controlled to correct the values around the estimated parameters. However, as the iterations increase, the step size is diverged. This is shown in Fig. 18(a), where $\phi_{e}$ starts to change its step size automatically after iteration 5.

This step size, through the use of exponents order $n$ given in (25), will contribute to how large the correction term $K_{i}$ will be at the next iteration and will contribute ultimately to update the interpolation factor adaptively. In particular, Fig. 18(b) shows two values of the exponents that have been chosen to investigate the performance of packet 6 over $1000 \mathrm{~m}$ channel range. In this figure, it can be seen that at $n=2$, the system exhibits an acceleration 


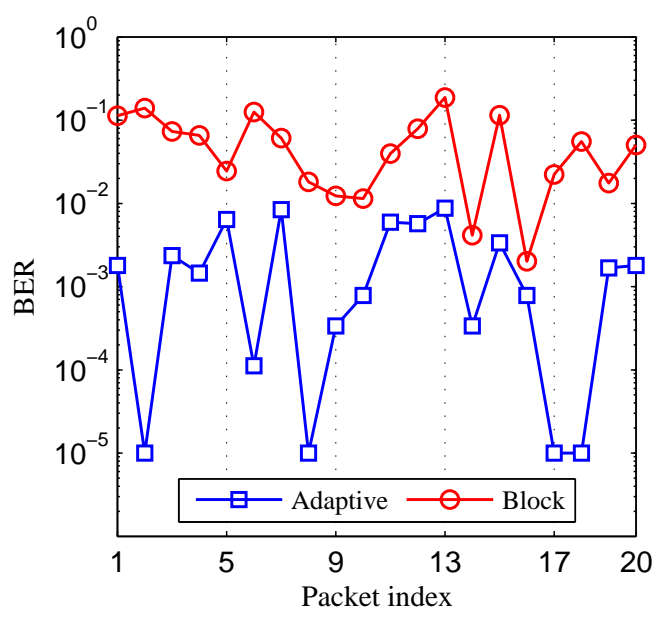

(a) $N_{c}=1024$.

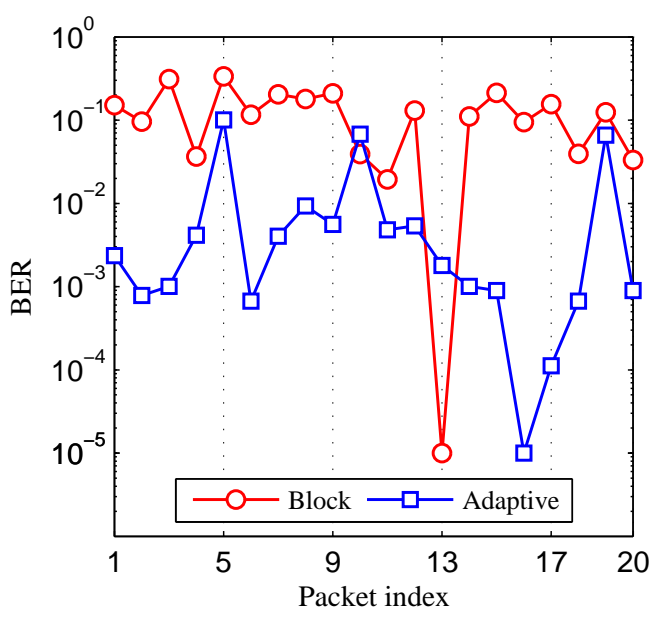

(b) $N_{c}=2048$.

Fig. 17. BER performance of adaptive time varying Doppler shift compensation receiver over $500 \mathrm{~m}$ channel range for different sub-carriers length, where the label $10^{-5}$ represents zero error.

of $\approx 1 \mathrm{~m} / \mathrm{s}^{2}$ due to the change of estimated speed, which is given as

$$
v_{r}(t)=[\Delta(t)-1] \times 1500
$$

during the symbol time that results in decoding errors in OFDM symbol 8 of packet 6 even with 10 inner iterations and 2 outer iterations. On the other hand, set $n=3$ demonstrates the speed is changing very smoothly during the symbol time and the acceleration is almost zero, therefore an evidence of the smoothness caused by the exponents order is interpreted in the resulting of zero error in packet 6 with 8 iterations as shown in Fig. 16(a). It is worth mentioning that no specific rule has been adopted to choose the exponents order $n$ in the proposed receiver. However, it has been noticed that the order increases proportionally with the sub-carriers, therefore, $n$ was set equal to 2, 3 and 4 for $N_{c}=512,1024$, and 2048, respectively. This is basically true on the assumption that it is likely the velocity changes with longer symbol time, especially with high acceleration, hence an adaptive step size is required.

The residual Doppler shift or CFO [19] destroys the orthogonality among sub-carrier frequency components. Consequently, in order to maintain the receiver performance, this CFO is estimated. As explained in Section III, a range of CFO candidates are chosen based on the sub-carrier spacing for each OFDM symbol length and a CFO that results in minimum phase error is selected among the range. Starting with $\epsilon=\Delta_{f} f_{c} T_{s}=0.25 \Delta_{f}$, where $f_{s}=1 / T_{s}=4 f_{c}$, and for each candidate $\epsilon_{i}$ repeat

$$
\hat{\epsilon}=\epsilon \epsilon_{i}
$$

where $\left\{\epsilon_{i} \in \mathbb{R}:-1 \leqslant \epsilon_{i} \leqslant 1\right\}$ for $N_{c}=1024$ and 2048 and $\left\{\epsilon_{i} \in \mathbb{R}:-2 \leqslant \epsilon_{i} \leqslant 2\right\}$ for $N_{c}=512$. To reduce the CFO search points, a step based on $\Delta_{f}$ was employed and can be formulated as $\Delta_{f} / 8$ in the case of $N_{c}=1024$ and 


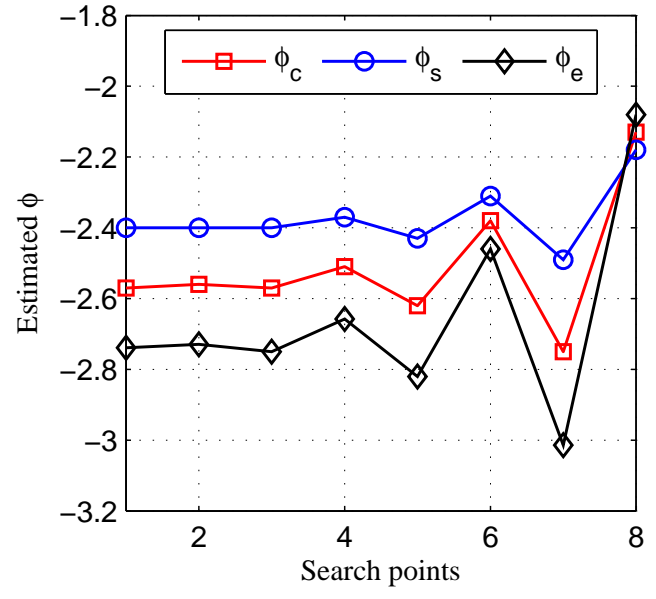

(a) Estimation of the parameter $\phi$ at $n=3$.

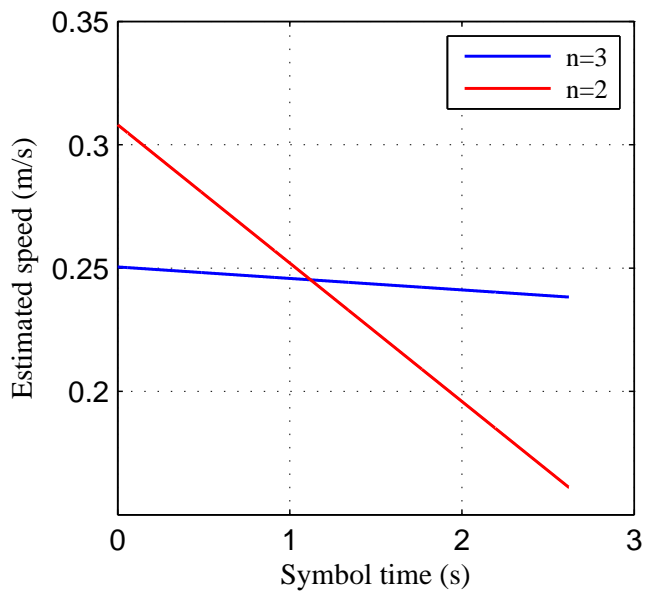

(b) Exponents effect on speed estimation of packet 6, symbol 8 .

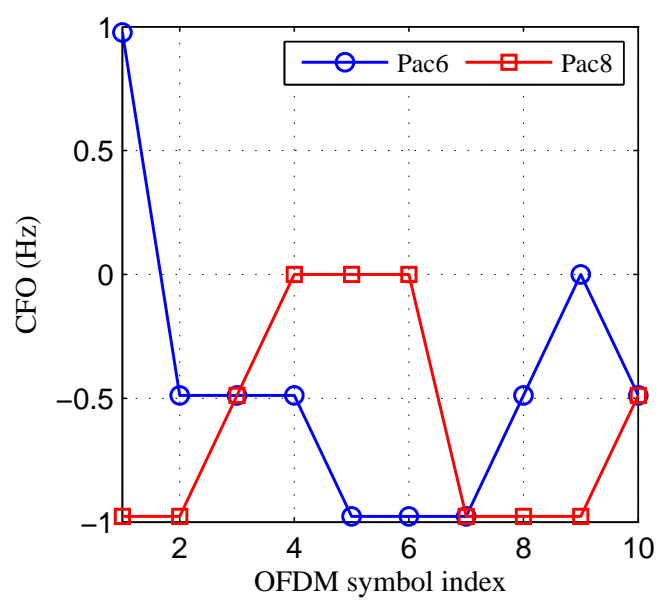

(c) CFO estimation for packets 6 and 8 .

Fig. 18. Effect of exponents on the estimation of $\mu_{i}$ and $K_{i}$, search points and smoothing the tracking step at $N_{c}=1024$ over $1000 \mathrm{~m}$ channel range.

2048, whereas the step was $\Delta_{f} / 16$ for $N_{c}=512$. Fig. 18(c) illustrates how the CFO changes within the duration of packets 6 and 8 . It can be inferred that there is no relation that governs the change in the residual Dopplershift between packet 6 and 8; the CFO changes randomly but sometimes constant. The residual Doppler-shift is depending on the accuracy of estimating the re-sampling factor.

\section{Conclusions}

The focus of this paper was on the Doppler effect caused by the acceleration due to the relative motion between the transmitter and receiver. This acceleration affects the correlation behaviour of the cyclic prefix and destroys the orthogonality of the sub-carriers due to the synchronization impairments. The proposed system is assessed through 
simulations at different scenarios and at different channel conditions to imitate the realistic case. Additionally, the suggested method is investigated with an off-line data that was recorded and processed from an experiment at the North Sea. This paper presented a technique to tackle the effect of a broadband time varying Doppler shift in UWC. This technique adopted a learning and punishment approach to iteratively estimate the Doppler shift parameters. These parameters were estimated by the cooperation of a two point estimation of the normalized correlation of the first order moment in addition to the linear prediction of the speed change. This method is very robust when the relative velocity is changing linearly and capable of dealing with the velocity inflection. The suggested algorithm is capable of tackling an acceleration up to $\pm 1 \mathrm{~m} / \mathrm{s}^{2}$ during the packet time and correcting a speed up to $3 \mathrm{~m} / \mathrm{s}$. 


\section{REFERENCES}

[1] A. F. Molisch, Wireless Communications. John Wiley, 2005.

[2] K. Tu, T. Duman, M. Stojanovic, and J. Proakis, " Multiple-resampling receiver design for OFDM over doppler-distorted underwater acoustic channels," IEEE J.Ocean. Eng., vol. 38, no. 2, pp. 333 - 346, Apr. 2013.

[3] J. Z. Huang, S. Zhou, and Z. H. Wang, " Performance results of two iterative receivers for distributed MIMO-OFDM with large Doppler deviations,” IEEE J.Ocean. Eng., vol. 38, no. 2, pp. 347 - 357, Apr. 2013.

[4] L. Wan, Z. Wang, S. Zhou, T. C. Yang and Z. Shi, "Performance comparison of doppler scale estimation methods for underwater acoustic OFDM,” Journal of Electrical and Computer Engineering, Article ID 703243, Jan. 2012.

[5] B. Li, S. Zhou, M. Stojanovic, L. Freitag, and P.Willett, "Multicarrier Communication Over Underwater Acoustic Channels With Nonuniform Doppler Shifts,"IEEE J.Ocean. Eng.,vol.33,no.2,pp. 198-209,Apr.2008.

[6] B. S. Sharif, J. Neasham, O. R. Hinton, and A. E. Adams, "A computationally efficient Doppler compensation system for underwater acoustic communications," IEEE J.Ocean. Eng.,vol.25,no.1,pp.52-61,Jan.2000.

[7] S. Mason, C. Berger, S. Zhou, and P. Willett, "Detection, Synchronization, and Doppler Scale Estimation with Multicarrier Waveforms in Underwater Acoustic Communication,"IEEE J. on Selected Areas in Communications, vol.26,no.9,pp. 1638-1649,Dec. 2008.

[8] J. van de Beek, M. Sandell and P.O. Borjesson, "ML estimation of time and frequency offset in OFDM systems," in Proc. IEEE Trans.Signal Process., vol. 45,no.7, pp.1800-1805, Jul. 1997.

[9] B. Kim and I. Lu., "Parameter study of OFDM underwater communications system," in Proc. IEEE Oceans, Providence, RI, vol.2, pp.12511255, Sep. 2000.

[10] A. B. Salberg, and A. Swami, “ Doppler and frequency-offset synchronization in wideband OFDM,"IEEE Trans. Wireless Commun.,vol.4,no.6,pp. 2870-2881,Nov.2005.

[11] A. Y. Kibangou, C. Siclet and L. Ros, "Joint channel and Doppler estimation for multicarrier underwater communications,"in Proc. IEEE ICASSP 2010, pp. 5630-5633, USA, March 14-19, 2010.

[12] Parrish et. al, "Symbol by symbol Doppler rate estimation for highly mobile underwater OFDM," Proc of WUWNET., November 2009.

[13] J. Armstrong, "Analysis of new and existing methods of reducing intercarrier interference due to carrier frequency offset in OFDM," IEEE Trans. Commun., vol. 47, no. 3, pp. 365369, Mar. 1999.

[14] A. F. Molisch, M. Toeltsch, and S. Vermani, "Iterative Methods for Cancellation of intercarrier interference in OFDM Systems," IEEE Transactions on Vehicular Technology, vol. 56, No.4, pp. 2158-2167, Jul. 2007.

[15] K. Tu, D. Fertonani, T. M. Duman and P. Hursky, "Mitigation of intercarrier interference in OFDM systems over underwater acoustic channels," in Proc. IEEE Ocean Conf., Bremen, pp. 1-6, 11-14 May 2009.

[16] S. Ahmed and H. Arslan, "Estimation and compensation of Doppler effect using variable sub-carrier spacing in multiband UAC OFDM systems," in Proc. IEEE Ocean Conf., Seattle, WA, pp. 1-4, 20-23 Sept. 2010.

[17] S. Yerramalli and U. Mitra, "Optimal resampling of OFDM signals for multiscalemultilag underwater acoustic channels,"IEEE J.Ocean. Eng., vol. 36, no.1, pp.126-138, Jan.2011.

[18] R. W. Bauml, R. F. H. Fisher, and J. B. Huber, "Reducing the peak-to-average power ratio of multicarrier modulation by selected mapping," IEE Electronics Letter, vol. 32, no. 22, pp. 20562057, Oct. 1996.

[19] M. Stojanovic, "Low-complexity OFDM detector for underwater channels,"in Proc. MTS/IEEE OCEAN Conf., Boston, MA, pp.18-21, Sep.2006.

[20] B. Li, S. Zhou, M. Stojanovic, L. Freitag and P. Willett, "Non-uniform Doppler compensation for zero-padded OFDM over fast-varying underwater acoustic channels,"in Proc. IEEE OCEAN 2007, Aberdeen, pp. 1 - 6, June, 2007.

[21] T. Pollet, P. Spruyt, and M. Moeneclaey, "The BER performance of OFDM systems using non-synchronized sampling,"in Proc.Global Telecommunications(GLOBECOM), vol.1,pp.253-257, Nov./Dec. 1994.

[22] M. Speth, S. Fechtel, G. Fock, and H. Meyr, "Optimum receiver design for wireless broad-band systems using OFDM-Part II," IEEE Trans. Commun., vol. 49, no. 4, pp. 571578, Apr. 2001.

[23] A. E. Abdelkareem, B. S. Sharif, C. C. Tsimenidis, J. A. Neasham and O. Hinton, "Low-complexity Doppler compensation for OFDM-based underwater acoustic communication systems," in Proc. IEEE Oceans, Santander, Spain, pp.1-6, Jun. 2011.

[24] Sanjita K. Mitra, Digital signal processing A computer based approach. McGraw-Hill, 2006. 
[25] A. E. Abdelkareem, B. S. Sharif, C. C. Tsimenidis and J. A. Neasham, "Time varying Doppler-shift compensation for OFDM-based shallow Underwater Acoustic Communication systems," in Proc. IEEE MASS, Spain, Valancia, pp. 885-891, Oct. 2011.

[26] Ali H. Sayed, Adaptive Filters. Wiley-IEEE Press, 2008.

[27] M. Abramowitz and I. A. Stegun, Handbook of Mathematical Functions with Formulas, Graphs, and Mathematical Tables. New York: Dover, 1972.

[28] W. Kim and D. C. Cox, "Residual Frequency Offset and Phase Compensation for OFDM Systems," in Proc. IEEE VTC, pp.2209-2213, 2007.

[29] R. Otnes and T. Jenserud and J. E. Voldhaug and C. Solberg, "A roadmap to ubiquitous underwater acoustic communications and networking," in Proc. 3rd UAM: Technologies and Results, Nafplion, Greece, Jun. 2009.

[30] C. Douillard, M. Jezequel, C. Berrou, A. Picart, P. Didier, and A. Glavieux, "Iterative correction of intersymbol interference: Turbo equalization,” European Trans. on Telecommun., vol. 6, pp. 507-511, Oct. 1995.

[31] A. Zielinski and Y. H. Yoon and L Wu, "Performance Analysis of Digital Acoustic Communication in a Shallow Water Channel,"IEEE J.Ocean. Eng., vol. 20, pp.293-299, Oct.1995. 